\title{
Magnetically-dominated jets inside collapsing stars as a model for gamma-ray bursts and supernova explosions
}

\author{
Dmitri A. Uzdensky* \\ Dept. of Astrophysical Sciences, Princeton University, \\ and Center for Magnetic Self-Organization (CMSO), Princeton, NJ 08544 \\ Andrew I. MacFadyen ${ }^{\dagger}$ \\ Dept. of Physics, New York University, and Institute for Advanced Study, Princeton, NJ 08540
}

(Dated: March 7, 2007)

\begin{abstract}
It has been suggested that magnetic fields play a dynamically-important role in core-collapse explosions of massive stars. In particular, they may be important in the collapsar scenario for gamma-ray bursts (GRB), where the central engine is a hyper-accreting black hole or a millisecond magnetar. The present paper is focussed on the magnetar scenario, with a specific emphasis on the interaction of the magnetar magnetosphere with the infalling stellar envelope. First, the "Pulsar-ina-Cavity" problem is introduced as a paradigm for a magnetar inside a collapsing star. The basic set-up of this fundamental plasma-physics problem is described, outlining its main features, and simple estimates are derived for the evolution of the magnetic field. In the context of a collapsing star, it is proposed that, at first, the ram pressure of the infalling plasma acts to confine the magnetosphere, enabling a gradual build-up of the magnetic pressure. At some point, the growing magnetic pressure overtakes the (decreasing) ram pressure of the gas, resulting in a magneticallydriven explosion. The explosion should be highly anisotropic, as the hoop-stress of the toroidal field, confined by the surrounding stellar matter, collimates the magnetically-dominated outflow into two beamed magnetic-tower jets. This creates a clean narrow channel for the escape of energy from the central engine through the star, as required for GRBs. In addition, the delayed onset of the collimated-explosion phase can explain the production of large quantities of Nickel-56, as suggested by the GRB-Supernova connection. Finally, the prospects for numerical simulations of this scenario are discussed.

PACS numbers: 52.25.Xz, 52.30.Cv, 95.30.Qd, 97.60.Bw, 97.60.Jd, 98.70.Rz
\end{abstract}

\section{INTRODUCTION}

Long-duration gamma-ray bursts (GRBs) are believed to be strongly asymmetric explosions of massive stars. Some explosion asymmetry has also been inferred in corecollapse supernova (SN) explosions. The asymmetry is expected if the progenitor star is rapidly rotating, with the rotation axis providing a preferred direction for a jetlike outflow.

The leading theoretical model for long-duration GRBs based on rapid stellar rotation the collapsar model [1, 2, 3]. In this model the core of a massive star collapses to form a black hole. If the star is rapidly rotating, the overlying stellar material can form an accretion disk around the black hole. Because of differential rotation in the disk, the magneto-rotational instability (MRI) is expected to develop (e.g., Ref. [4]), providing angular momentum transport and dissipation of gravitational energy. At the temperatures and densities $\left(T \sim 4 \times 10^{10}\right.$ $\mathrm{K}, \rho \sim 10^{10} \mathrm{~g} \mathrm{~cm}^{-3}$ ) present in collapsar disks, neutrino emission cools the gas and the collapsing outer stellar core accretes at rates of $\sim 0.1 M_{\odot} \mathrm{s}^{-1}$ for times $\gtrsim 10 \mathrm{sec}$, long enough for the jet to break out of the star and to power long GRBs. This accretion disk-black hole system thus acts as the central engine for the GRB. The energy source in this case is mainly accretion. Since most of this energy is released via neutrinos, most models have relied on neutrino luminosity to power the outflow. However, it has also been suggested that a significant amount of energy may be extracted magnetically and that strongly magnetized jets may play an active role in GRB explosions [4, 5, 6, 7, 8, 9, 10, 11, 12, 13, 14, 15, 16, 17, 18]).

In order for magnetic mechanisms to be viable, a fairly strong fields (of order $10^{15} \mathrm{G}$ ) are required. Such fields, however, are expected to be generated by the MRI-driven turbulent dynamo in the disk during core-collapse [4]. Similar processes, resulting in similar field strengths, are also believed to be taking place in core-collapse supernovae explosions [19, 20]. For example, Akiyama \& Wheeler have argued [19] that the field may reach the level set by the equipartition with the MRI-driven turbulence, as strong as $10^{16-17} \mathrm{G}$. However, as they point out, this field is mostly toroidal. The large-scale poloidal magnetic field that is needed may require a large-scale helical dynamo 21] and will probably be somewhat smaller than the toroidal field. Thus, we believe it is not unreasonable for the poloidal field at the disk surface to be a more modest $B_{d} \sim 10^{15} \mathrm{G}$ (see also Ref. [4]).

In this paper we focus mostly on an alternative scenario, in which the central engine operating inside a collapsing star is not a black hole but a young millisecond magnetar - a neutron star (NS) with a large-scale 
poloidal magnetic field of the order of $10^{15}$ G. Such a strong magnetic field can be produced by a convectively driven turbulent dynamo 22, 23 inside the young NS, or by the MRI-driven dynamo in the differentially rotating collapsing core [19]. An alternative possibility is that the progenitor core of about $10^{4} \mathrm{~km}$ has a magnetic field of order $10^{9} \mathrm{G}$, similar to the fields observed in some white dwarfs. When such a highly-magnetized core collapses into a neutron star of $10 \mathrm{~km}$ radius, flux freezing leads to the amplification of the magnetic field to $10^{15} \mathrm{G}$ (e.g., Ref. [15]).

We see that the typical values of the magnetic field strength, the rotation rate, and the size of the central engine in the classical collapsar scenario with an accreting black hole and in the millisecond-magnetar case are similar. The overall electro-magnetic luminosities should therefore be also comparable. And indeed, for a typical surface magnetic field $B_{*}=10^{15} \mathrm{G}$, a rotation rate $\Omega_{*}=10^{4} \mathrm{sec}^{-1}$, and a radius $R_{*}=10 \mathrm{~km}$, the basic energetics and timescales make the millisecond magnetar a plausible candidate for a GRB central engine [5]. Ultimately, the energy source for the explosion is the rotational energy of the neutron star and the magnetic field acts as the agent that extracts this energy. In particular, the rotational energy of a millisecond-period neutron star is of order $E_{\text {rot }} \simeq 5 \cdot 10^{52} \mathrm{erg}$, more than enough to drive a long-duration GRB. The energy-extraction time scale, estimated using the usual pulsar luminosity formula $L_{\text {magn }} \sim B_{*}^{2} R_{*}^{6} \Omega_{*}^{4} c^{-3}$, is then of order $100 \mathrm{sec}$. Thus, the millisecond-magnetar central engine is essentially similar (with the magnetic field scaled up by three orders of magnitude and the timescale scaled down by six) to the Ostriker \& Gunn model 24] for powering supernovae by the spin-down magnetic power of a rapidly rotating pulsar.

The idea of a millisecond magnetar as a central engine for gamma-ray bursts has been first proposed independently by Usov 25] and by Duncan \& Thompson 22. It has been further developed by a number of authors 5,26 , 27, 28, 29, 30, 31, 32, 33]. In addition, several magnetic explosion mechanisms have been explored in the supernova context 19, 20, 21, 30, 31, 33, 34, 35, 36, 37, 38, 39]). Our present paper is also devoted to investigating the millisecond-magnetar scenario for GRBs, but, importantly, viewed within the overall context of a collapsing star.

First we would like to stress that the plausible overall energetics and timescales are, by themselves, not sufficient for making a good GRB central engine model. The central engine also needs to be able to produce an energetic outflow that is (1) ultra-relativistic; (2) highlycollimated; and (3) baryon-free. Most of the previous studies have focused mostly on the energetics and timescales, but not on the mechanisms for producing an outflow that satisfies these requirements (see, however, Refs. [30] and [37] for a discussion of collimation).
Also, most of these models, with the notable exception of Refs. [30] and [40], have considered a magnetar in isolation, ignoring the effect of the surrounding stellar gas on shaping the outflow.

One of the major points of our paper is that the infalling stellar gas is still present during the explosion and needs to be taken into account. Thus, an important new element distinguishing our model from previous works is the consideration of the interaction between a newly-born magnetar and the stellar plasma in which it is initially embedded. Specifically, we argue that the pressure and inertia of the surrounding gas play a key role in regulating the magnetic extraction of rotational energy from the magnetar. They also force the Poynting-flux-dominated outflow into two collimated jets, similar to Lynden-Bell's magnetic towers 15, 41, 42].

In Section a we discuss a specific situation relevant to the core collapse of a massive star: a cavity inside the stalled bounce-shock. The radius of such shock stays roughly constant allowing the magnetic fields in the cavity to grow. At the same time, both the ram pressure of the infalling gas and the neutrino energy deposition inside the cavity decrease with time. We therefore argue that at some point, a fraction of a second after bounce, the magnetic field will start to dominate the force balance, leading to a magnetically-driven explosion.

In order to illustrate these ideas, we introduce the "Pulsar-in-a-Cavity" problem as a basic-physics paradigm for this scenario. We describe this idealized problem in detail in Section . We first give a general description of the problem and its several versions. In Section . we consider the simplest case of a force-free rotating magnetosphere inside a fixed rigid cavity. We demonstrate that differential rotation of the magnetic field lines is inevitably established inside the cavity, even though the pulsar itself is rotating uniformly; as a result, a strong toroidal magnetic field gradually builds up. We then study the long-term evolution of the field inside the cavity and show that the magnetic luminosity increases with time. We also show that a massive, non-force-free plasma strip unavoidably arises in the equatorial plane beyond the light cylinder. We expect this phase to last until either the development of the kink instability modifies the situation or until the cavity walls yield to the internal magnetic stresses. In Section ${ }_{\square}$ we discuss hoopstress collimation and argue that external confinement and differential rotation are two important ingredients for collimating relativistic Poynting-flux dominated outflows. In either case, a magnetic tower forms.

We investigate the propagation of a magnetic tower through the star in Section ${ }_{\square}$ Section ${ }_{a}$ is devoted to a general description of Lynden-Bell's magnetic tower model[41], whereas Sections ${ }_{\square}{ }_{\square}{ }_{\square}$ describe our modification of this model for the stellar environment. Specifically, we suggest that the external confining pressure invoked by Lynden-Bell's model is provided by the gas in- 
side a hot cocoon behind a strong shock that the rapidlygrowing magnetic tower drives into the unperturbed stellar envelope.

In Section w we further explore some of the astrophysically-interesting aspects of our model. In Section \& we consider the transition of the magnetic tower expansion to the relativistic regime and the final opening angle of the tower. In Section \& we discuss a possible small-scale substructure of the magnetic tower, represented as a "train of plasmoids", that may be the outcome of the small-scale magnetic structure at the base of the outflow (e.g., in the magnetized corona of the magnetar or of the accretion disk); it may also develop later as a result of MHD instabilities and flux conversion in the growing magnetic tower itself. In Section $\square$ we discuss these stability issues, especially in regard to the Rayleigh-Taylor and kink instabilities. In Section 口 we investigate the prospects for reconnection in the magnetar magnetosphere or in the magnetic tower, and argue that reconnection is ineffective in the dense environment deep inside the collapsing star. In Section $\square$ we address an important issue of ${ }^{56} \mathrm{Ni}$ production and argue that the two-phase nature of the explosion in our model is wellsuited to explain a large amount of ${ }^{56} \mathrm{Ni}$ inferred from observations. In Section ${ }_{a}$ we discuss the implications of our model for pulsar kicks. Finally, in Section 口 we suggest some directions for future numerical simulations of this problem. We draw our conclusions in Section

\section{MAGNETAR INSIDE A COLLAPSING STAR: AN OUTLINE OF THE GENERAL SCENARIO}

Numerous studies of core-collapse supernovae have shown that, as the core of a massive star collapses into a proto-neutron star (PNS), a bounce shock is launched back into the star but quickly stalls at about $200 \mathrm{~km}$ (see, e.g., Refs. [43, 44]). The explosion then enters a long $(\sim 1 \mathrm{sec})$ phase (see Fig. 1) during which the shock is quasi-stationary as determined by the balance between the ram pressure of the infalling material, which tends to quench the shock, and the thermal pressure of the post-shocked gas, supported by the continuous neutrino heating. Eventually, if neutrinos win, the shock engulfs the entire star and one gets a successful SN explosion. If they lose, the shock dies and the PNS collapses into a black hole that subsequently swallows the rest of the star, without a SN.

In our model, we add a third dynamical component to this picture - the magnetic field. The magnetic pressure is pushing out, helping the explosion, as is the thermal pressure of the neutrino-heated gas. Our main idea is that these two outward forces evolve differently with time, and thus the explosion may be a two-stage process. In particular, we suggest that the magnetic pressure is not important during the first few hundreds of msec of the stalled-shock phase. However, during this time the magnetar makes several hundred revolutions. This results in a great amplification of the toroidal magnetic flux by the differential rotation (see Sec. $\mathrm{a}$ ), whereas both the neutrino energy deposition and the accretion rate gradually decline (see Fig. 21). For example, assuming $R_{0}=3 R_{\mathrm{LC}}=10 R_{*}=100 \mathrm{~km}$, and $B_{*}=10^{15} \mathrm{G}$, the entire cavity is filled (see Sec. ${ }_{\text {a }}$ ) with $3 \cdot 10^{14} \mathrm{G}$ fields after about 100 turns $(0.1 \mathrm{sec})$, corresponding to the magnetic pressure of $4 \cdot 10^{27} \mathrm{erg} / \mathrm{cm}^{3}$. The ram pressure of the infalling stellar material at $r=R_{0}$, compressing the magnetosphere, can be estimated roughly as $P_{\mathrm{ram}} \sim \dot{M} v_{\mathrm{ff}} / 4 \pi R_{0}^{2} \simeq 8 \cdot 10^{27} \dot{M}_{0} M_{0}^{1 / 2} R_{0,7}^{-5 / 2} \mathrm{erg} \mathrm{cm}^{-3}$ where $v_{\text {ff }}=\left(2 G M / R_{0}\right)^{1 / 2} \simeq 5 \cdot 10^{9} M_{0}^{1 / 2} R_{0,7}^{-1 / 2} \mathrm{~cm} / \mathrm{sec}$ is the free-fall velocity at radius $R_{0}$, and $M_{0}$ and $\dot{M}_{0}$ are the mass within $R_{0}$ and the accretion rate at this radius, expressed in units of $M_{\odot}$ and $M_{\odot} /$ sec, respectively. This shows that, after a delay of a few hundreds of milliseconds, the magnetic pressure inevitably becomes an important driving force and may lead to a successful explosion.

To summarize our picture, the ram pressure of the accreting material provides a nurturing womb in which the baby magnetic field grows, until it is finally strong enough to break out. Neutrino energy deposition plays an important role during this gestation period, as it prevents the magnetosphere from being completely squashed by the accreting gas. Finally, if the above picture is correct and the explosion does become magnetically-driven, then the hoop-stress mechanism makes it highly collimated, thus satisfying one of the key necessary conditions for GRB (see Sec. ${ }_{0}$ ). Note that this jet is driven by the magnetar-level (i.e., $\sim 10^{15} \mathrm{G}$ ) field and is thus much stronger and faster than the LeBlanc-Wilson jet 34 that may have been launched a few seconds earlier, during the core-collapse process [30].

\section{THE PULSAR-IN-A-CAVITY PROBLEM}

As a first step in trying to understand how a millisecond-magnetar central engine works in the collapsar context, let us consider the following basic plasmaphysics problem: an axisymmetric pulsar inside a conducting cavity with a low-density plasma (see Fig. 3). Specifically, we consider the cavity's radius $R_{0}$ to be is much larger than the pulsar light-cylinder radius $R_{\mathrm{LC}}$. We call this idealized problem the Pulsar-in-a-Cavity problem [15, 32]. It is a modification of the Goldreich \& Julian model for an isolated pulsar's magnetosphere [45]; it has direct connections to the model proposed by Ostriker \& Gunn for powering longer-term supernova lightcurves [24] and it is also related to the models considered by Kardashev [46] and by Illarionov \& Sunyaev 47].

Note that the behavior of Pulsar-in-a-Cavity depends 
on the cavity properties. For definiteness, we assume that both the walls and the plasma inside the cavity are perfect conductors. We also assume that all the field lines close back to the pulsar inside the cavity, keeping in mind that the magnetic field had been generated inside the NS and then emerged through its surface.

At the same time, we are dealing with a whole family of problems distinguished by the assumed mechanical properties of the cavity. For simplicity, we shall concentrate on the case of a spherical cavity with rigid walls (see Sec. $)$, which may correspond to early stages of the system's evolution. In the future we plan to consider more realistic but also more complicated cases where the shape and the size of the cavity are not fixed but instead are governed by a balance with an external pressure (similar to Lynden-Bell's magnetic tower model 41]), and, finally, will consider a young pulsar in a fully dynamic environment of a collapsing star (c.f., Ref. [24]).

Each of these versions of our pulsar-in-a-cavity problem will provide important insights into the workings of a millisecond magnetar inside a collapsing star. They will probably require numerical simulations using relativistic force-free or relativistic MHD codes (see Sec. „). To set the stage for these numerical studies, we will, in this section, qualitatively discuss a plausible physical picture of the system's evolution.

To investigate the interaction between the central magnetar and the surrounding stellar material, a full magnetohydrodynamic (MHD) description that includes plasma pressure and inertial effects will eventually be required. This is especially relevant if there is a strong wind driven off the PNS by neutrinos and/or by the magneto-centrifugal mechanism (e.g., Refs. [33, 37, 38]). Of particular interest is the confinement of the expanding magnetosphere by the surrounding plasma and the dynamical response of the star to the expanding magnetosphere at its center. For simplicity, however, here we shall limit ourselves to the relativistic force-free case in which electromagnetic forces dominate the dynamics almost everywhere inside the cavity (except at the the equatorial plane outside the light cylinder - see below). While it is not realistic in the central region of a collapsing star, the force-free description may nonetheless reflect some essential physics, especially for late phases of the evolution.

We would like to remark that the ideal-MHD assumption is well justified due to the very large plasma densities and temperatures. Specifically, the high plasma density ensures that the plasma is highly collisional and hence is well described by resistive MHD; other non-ideal terms in generalized Ohm's law are unimportant. On the other hand, the resistivity is actually small in absolute terms $\left(R e_{m} \gg 1\right)$. All this makes ideal MHD a good approximation in the environment of a collapsing star [15]. Therefore, in this paper we shall ignore any non-ideal effects, leaving them for a future study.
As a final point, in contrast with the isolated pulsar, which is usually regarded as stationary, our pulsar-in-acavity problem is intrinsically time-dependent.

\section{Pulsar in a Fixed Spherical Cavity}

In this section we consider the case of a spherical cavity whose radius, $R_{0}$, is kept constant (or is changing slowly). We are interested in the evolution of an initially dipole-like magnetosphere after the pulsar is spun up suddenly. In the isolated pulsar case, the field lines extending beyond the light cylinder are swept backwards and open [45]; there is no feedback of the outer region on the inner magnetosphere. In our case, however, the entire magnetosphere is contained inside the cavity and hence remain closed at all times. This is a very important difference between the two cases.

The first thing to note is that a differential rotation is established in the magnetosphere. Indeed, consider a field line $\Psi$ that extends beyond the light cylinder (Fig. 33) and compare the angular velocities at two points: point $A$ where this line attaches to the pulsar and point $B$ where it intersects the equator. The angular velocity at point $A$ is clearly equal to that of the neutron star: $\Omega_{A}=\Omega_{*}$. Next, what is the angular velocity at point $B$ ? Since the field line in question extends beyond the light cylinder, it cannot remain purely poloidal and a toroidal field has to develop. Due to the assumed symmetry with respect to the equator, however, the toroidal field has to vanish at $z=0, B_{\phi}(z=0) \equiv 0$, and the plasma cannot slide toroidally backward along the field. Therefore, $\Omega_{B}$ has to equal the angular velocity of the plasma at this point. Now, the toroidal field that develops in the magnetosphere off the equatorial plane continuously brakes the star down, so that there is an outward flux of angular momentum and a Poynting flux of energy along the field line. The rotational energy of the pulsar extracted by the magnetic field is partly accumulated in the magnetic form and partly transferred to the equatorial plasma. Thus, the material at point $B$ is continuously torqued up by the magnetic field. Then, since the confining wall prevents the material from moving out freely in the radial direction, the toroidal velocity of the plasma becomes closer and closer to the speed of light. However, it can never exceed the speed of light; therefore, the angular velocity at point $B$ is bounded: $\Omega_{B} \simeq c / R_{B}=\Omega_{*} R_{\mathrm{LC}} / R_{B}$. Thus, we see that the field line experiences differential rotation at a rate $\Delta \Omega=\Omega_{A}-\Omega_{B} \geq \Omega_{*}\left(1-R_{\mathrm{LC}} / R_{B}\right)$; For field lines with $R_{B} \gg R_{\mathrm{LC}}$ we get $\Delta \Omega \approx \Omega_{*}$. This differential rotation is established on the cavity's light-crossing time-scale, $t_{0} \equiv R_{0} / c \gg \Omega_{*}^{-1}$.

This differential rotation is important because it leads to a continuous injection of toroidal magnetic flux (of opposite signs) into the upper and lower hemispheres. If the cavity's size is fixed, then the toroidal magnetic field 
at any point grows roughly linearly with time. This is in sharp contrast with the unbounded pulsar case in whcih a steady state is established on the light travel time-scale.

Let us now analyze the field evolution on long time scales $\left(t \gg t_{0} \equiv R_{0} / c\right)$ and at distances $R \gg R_{\mathrm{LC}}$. Note that the toroidal magnetic field continuously increases, whereas the poloidal magnetic field does not. The poloidal electric field, $E_{\mathrm{pol}}$, may become much larger than $B_{\text {pol }}$ but in any case cannot exceed the value $B_{\mathrm{pol}} \Omega_{*} R_{0} / c=B_{\mathrm{pol}} R_{0} / R_{\mathrm{LC}}$. Thus, after several light-crossing times $\left(t \gg t_{0}\right)$ the magnetosphere outside the light cylinder is dominated by the toroidal field, $B_{\phi} \gg E_{\mathrm{pol}}, B_{\mathrm{pol}}$.

Next, since $B_{\phi} \sim t$, the relative change in $B_{\phi}$ over $\Delta t \sim$ $t_{0}$ becomes small at late times, $t \gg t_{0}$. An approximate force-free equilibrium is then established in each hemisphere, described by the relativistic force-free $\mathrm{Grad}-$ Shafranov equation. In the $B_{\phi}$-dominated limit this equation reduces to $I I^{\prime}(\Psi)=0$, where $I \equiv R B_{\phi}$ is the enclosed poloidal current. The obvious solution of this equation is $I(\Psi)=I_{0}=$ const, i.e., a singular line current $I_{0}(t)$ along the rotation axis. The toroidal magnetic field is the vacuum field produced by this line current, $B_{\phi}(t, R, Z)=I_{0}(t) / R$; i.e., constant on cylinders. The main force balance in the magnetosphere is between the toroidal field tension and pressure. In other words, the poloidal current becomes spatially separated from the toroidal magnetic field: it flows out of the pulsar along the axis (in both hemispheres), then as a surface current along the cavity walls, and finally returns to the pulsar along the non-force-free equatorial current sheet present due to the sharp reversal of the toroidal field across the equator. The current density in the bulk of the magnetosphere is relatively small. This is similar to the electric current structure of the magnetic bubble considered by Lyutikov \& Blandford [11].

We shall express magnetic quantities characterizing the field in the cavity in terms of the total poloidal magnetic flux $\Psi_{0}$ extending beyond the light cylinder. This flux can be crudely estimated from the pure dipole magnetic field, i.e.,

$$
\Psi_{0} \sim \Psi_{\text {dipole }}\left(R_{\mathrm{LC}}\right)=B_{*} \frac{R_{*}^{3}}{R_{\mathrm{LC}}} .
$$

Then, the characteristic poloidal magnetic field strength in the cavity at distances $r \sim R_{0}$ from the center and off the equatorial plane can be estimated as

$$
B_{\mathrm{pol}} \sim B_{0} \equiv \frac{\Psi_{0}}{R_{0}{ }^{2}} \sim B_{*} \frac{R_{*}^{3}}{R_{0}^{2} R_{\mathrm{LC}}} .
$$

Let us now estimate the poloidal line current $I_{0}(t)$ and hence the characteristic toroidal field in the cavity. The poloidal current is found by following the shape of a field line $\Psi$ :

$$
I(\Psi, t)=\Delta \Omega t\left[\int_{\Psi} \frac{d l_{\mathrm{pol}}}{B_{\mathrm{pol}} R^{2}\left(l_{\mathrm{pol}}\right)}\right]^{-1},
$$

where $l_{\text {pol }}$ is the path-length along the poloidal field. The main contribution comes from large distances, $R \sim R_{0}$, and thus, using $\Delta \Omega \simeq \Omega_{*}=c / R_{\mathrm{LC}}$, we get

$$
I_{0}(t) \sim \Omega_{*} t \frac{\Psi_{0}}{R_{0}} \simeq \frac{\Psi_{0}}{R_{\mathrm{LC}}} \frac{t}{t_{0}} .
$$

We see that for $t \gg t_{0}$ the poloidal current becomes much stronger than that in the isolated pulsar magnetosphere $\left(I \sim \Psi_{0} / R_{\mathrm{LC}}\right)$. Using the estimate (11) for $\Psi_{0}$, we obtain

$$
I_{0}(t) \sim B_{*} \frac{R_{*}^{3}}{R_{\mathrm{LC}}^{2}} \frac{t}{t_{0}} .
$$

Correspondingly, the characteristic toroidal magnetic field at distances of order $R_{0}$ is

$$
B_{\phi}\left(R_{0}\right)=\frac{I_{0}}{R_{0}} \simeq B_{0} \Omega_{*} t,
$$

which is similar to the estimate presented by Kardashev for the toroidal field of a pulsar inside an expanding supernova cavity [46]. Thus, after many light-crossing times across the cavity, $B_{\phi}\left(R_{0}\right)$ becomes much larger than the toroidal field of an isolated pulsar at these distances $\left[B_{\phi}^{\text {isolated }} \sim \Psi_{0} /\left(R_{0} R_{\mathrm{LC}}\right)=B_{0}\left(R_{0} / R_{\mathrm{LC}}\right)=B_{0} \Omega_{*} t_{0} \ll\right.$ $\left.B_{0} \Omega_{*} t\right]$.

As we noted above, the magnetosphere outside the pulsar light cylinder cannot be entirely force-free. Because the toroidal magnetic field reverses across the equator, the magnetic tension continuously accelerates the equatorial plasma in the toroidal direction. The tension force performs mechanical work on the equatorial plasma and so a part of the rotational energy extracted from the pulsar is deposited in the equatorial plane (the rest is stored magnetically in the bulk of the cavity). Since the plasma in the equatorial plane rotates ultra-relativistically, the added energy leads to an increase in the relativistic "mass" of the plasma, $\Delta m \sim t^{2}$. Correspondingly, this relativistically rotating massive equatorial sheet experiences an outward centrifugal force, $F_{\text {cent }}$. This force cannot be balanced by the toroidal magnetic field because the latter is zero at the equator and so the equatorial plasma moves out towards the wall. It then pushes the poloidal magnetic flux out and concentrates it a narrow equatorial strip of ever-decreasing width $d(t) \ll R_{0}$ near the wall (see Fig. (4). Because of this, nearly all the poloidal flux $\Psi_{0}$ that extends beyond the light cylinder crosses the equator at cylindrical radii $R \simeq R_{0}$. At the same time, in the magnetosphere above and below the equatorial plane, the poloidal field lines that emanate from this strip fan out to fill the cavity volume. Thus, the characteristic poloidal magnetic field in the cavity is 
of the order $B_{0}=\Psi_{0} / R_{0}^{2}$ (see eqn. 21) and is much weaker (by a factor of $d / R_{0}$ ) than in the equatorial strip.

Let us assess the centrifugal force quantitatively. The total torque on the massive equatorial strip is $\tau(t)=$ $\int I(\Psi, t) d \Psi \simeq I_{0}(t) \Psi_{0}$ and the total work per unit time (the total Poynting flux coming to the strip) is $P_{\text {strip }} \simeq$ $\tau c / R_{0}=B_{\phi} c \Psi_{0}$. This power goes into accelerating the plasma rotation, that is, into increasing the rotational $\gamma$-factor and hence the relativistic mass $m$ of the plasma in the strip:

$$
m(t) c^{2}=P t \sim\left(\frac{t}{t_{0}}\right)^{2} \frac{R_{0}}{R_{\mathrm{LC}}} \frac{\Psi_{0}^{2}}{R_{0}} \sim \frac{R_{\mathrm{LC}}}{R_{0}} B_{\phi}^{2}(t) R_{0}^{3} .
$$

That is, the kinetic energy in the equatorial strip is always small compared with the magnetic energy in the cavity, $B_{\phi}^{2}(t) R_{0}^{3}$. The centrifugal force acting on the equatorial strip is

$$
F_{\text {cent }}(t)=\frac{m(t) c^{2}}{R_{0}} \sim B_{0}^{2} R_{0}^{2} \Omega_{*}^{2} t^{2} \frac{R_{\mathrm{LC}}}{R_{0}} \sim B_{\phi}^{2}(t) R_{0}^{2}\left(\frac{R_{\mathrm{LC}}}{R_{0}}\right) .
$$

This force grows quadratically with time, just as the toroidal field pressure, but always remains small (by a factor of $R_{\mathrm{LC}} / R_{0} \ll 1$ ) compared with the total horizontal force exerted on the side wall by the toroidal field. However, since $F_{\text {cent }}$ is concentrated in the thin equatorial region, it may be important in a subsequent expansion of the cavity (c.f. non-relativistic MHD simulations by Matt et al., Ref. [48]).

A detailed analysis of the internal structure of the massive equatorial plasma strip is an interesting problem that should be studied but it lies beyond the scope of this paper.

Another very important point is that the rate at which the magnetic field in a confined magnetosphere extracts rotational energy from the central rotating conductor actually grows with time. This is because the magnetic torque per unit area is proportional to the toroidal field at the conductor's surface and the latter grows linearly with time. Thus, as long as the cavity does not expand (or expands slowly) and the rotation rate of the spinning pulsar stays constant, the magnetic power generated by the pulsar inside a cavity increases linearly with time:

$$
P(t)=I(t) \Psi_{0} \Omega_{*}=\Omega_{*}^{2} t \frac{\Psi_{0}^{2}}{R_{0}} \sim P_{\text {isolated }} \frac{c t}{R_{0}},
$$

where $P_{\text {isolated }} \sim B_{*}^{2} R_{*}^{6} \Omega_{*}^{4} / c^{3}$ is the spin-down power of an isolated, unbounded pulsar. Hence, after many lightcrossing times, the power of a pulsar-in-a-cavity greatly exceeds that of a classical isolated pulsar. This is resolves the apparent paradox raised by Lyutikov [13].

This runaway behavior can be attributed to a positive feedback between the energy that has been already extracted from the pulsar, and the strength of the agent that extracts the energy (the toroidal magnetic field).
Namely, most of the extracted energy is stored in the toroidal magnetic field, and, since the volume is finite, the toroidal field strength increases with time. Since the magnetosphere remains in a quasi-equilibrium, the toroidal field constantly readjusts everywhere and the inner magnetosphere feels the presence of the outer confining wall. In particular, the toroidal field at the pulsar surface increases linearly with time, and so does the magnetic spin-down torque on the pulsar. This picture is similar to the combustion chamber of a rocket. In that case, the gas temperature and pressure increase as the chemical energy of the fuel is released in the combustion process. At the same time, the fuel burning rate grows with the ambient temperature. Therefore, a rapid and efficient burning demands high pressure and is hence facilitated by a strong confining chamber. Similarly, in our case of a pulsar placed inside a cavity, the presence of strong cavity walls leads to an increased energy extraction rate from the pulsar.

In reality, we don't expect this power growth to last indefinitely. It may saturate, for example, due to the development of the kink instability, resulting in the conversion of the toroidal flux to poloidal flux and to the dissipation of some of the magnetic energy (see Sec. ${ }$ for more discussion).

\section{Hoop-stress collimation: contrast with the isolated pulsar}

The toroidal field generated by the differential rotation exerts a constantly-growing pressure on the cavity walls. If we now relax the fixed-wall assumption, this pressure will inflate the cavity. Will this inflation be isotropic or, say, collimated along the axis?

Generally speaking, since the toroidal field pressure in the horizontal direction is partly negated by the field's tension, one expects the resulting expansion to be mostly vertical. However, the differential rotation producing $B_{\phi}$ is relativistic: $\Delta \Omega R_{0} \sim \Omega_{*} R_{0} \gg c$, and it is well-known that hoop-stress collimation is not a trivial issue in the relativistic case. Thus, it is not immediately obvious this mechanism can be applied to our pulsar-in-a-cavity scenario. The quintessential example of this lack of collimation for ultra-relativistic magnetically-dominated outflows is the isolated aligned pulsar wind inside the termination shock. The basic reason for this is the decollimating force due to the poloidal electric field, $E_{\mathrm{pol}}$. Indeed, in the case of an unbounded relativistic uniformlyrotating force-free magnetosphere in a steady state, $E_{\text {pol }}$ and $B_{\phi}$ are nearly equal at large distances from the axis 45]. Importantly, this balance happens in an uncollimated, quasi-spherical poloidal magnetic field configuration; an excellent example of this is Michel's splitmonopole solution [49]. Here is a crude argument explaining this lack of hoop-stress collimation in the relativistic- 
rotation case. Consider an uncollimated field configuration; the poloidal magnetic field is open outside the light cylinder and has a split-monopole geometry, i.e., drops off as $r^{-2}$. In a steady state, the poloidal electric field is $E_{\mathrm{pol}}=B_{\mathrm{pol}} R / R_{\mathrm{LC}}$, where $R$ is the cylindrical radius; it hence drops off along radial rays as $r^{-1}$. But $B_{\phi}$ also drops off as $r^{-1}$. Moreover, at the light cylinder, $E_{\mathrm{pol}}=B_{\mathrm{pol}} \sim B_{\phi}$. Since outside the light cylinder they both decrease as $r^{-1}$, they remain comparable to each other at large distances. In fact, as Goldreich \& Julian showed 45], $E_{\text {pol }}$ and $B_{\phi}$ become equal asymptotically as $r \rightarrow \infty$. The bottom line is that a quasi-spherical relativistic force-free equilibrium can be established as a balance between the collimating pinch force (the sum of the toroidal magnetic field pressure and its tension) and the opposing electric force. Hoop-stress collimation is suppressed as a result of this balance.

On the other hand, the case of a rotating magnetosphere enclosed inside a cavity is different and hoop-stress collimation can in fact work. Indeed, as we showed above, at late times the toroidal magnetic field filling the cavity becomes stronger than both $B_{\mathrm{pol}}$ and $E_{\mathrm{pol}}$, in contrast to the isolated pulsar case. Furthermore, this toroidal field is distributed nonuniformly; namely, $B_{\phi} \sim R^{-1}$. Correspondingly, the magnetic pressure pushing vertically against the top and bottom walls is much higher than that on the side walls. Therefore, if we now allow the cavity to expand under this pressure, the expansion will be mostly vertical. The situation is then similar to the non-relativistic magnetic tower proposed by Lynden-Bell[41]. We therefore envision that long-term result will be the creation of a pair of oppositely-directed magnetic towers [15]. The interaction of the expanding towers with the surrounding stellar envelope aids in their confinement, similarly to jet collimation in hydrodynamical simulations of the collapsar model $[3,50,51]$. In the scenario considered here, the towers are driven not by a differentially-rotating disk, but by a rapidly-rotating magnetar. This suggests that considering the pulsar magnetosphere inside a cylindrical, as opposed to spherical, cavity may represent yet another interesting topic for future research.

An important element in the above discussion is the fact that $E_{\mathrm{pol}}$ is small compared with $B_{\phi}$. This is because $B_{\phi}$ is generated as a result of differential rotation. This highlights the important role of differential rotation (as opposed to uniform relativistic rotation) in collimating relativistic force-free outflows.

\section{MAGNETIC TOWER INSIDE A STAR}

For simplicity, in this section we shall mostly consider the case when the central engine is an accretion disk around a black hole. However, we believe that the millisecond-magnetar case is essentially similar.

\section{Lynden-Bell's Original Magnetic Tower Model}

We suggest that the model most naturally suited to describe the propagation of a Poynting-flux dominated jet through a star is the magnetic tower model, originally introduced by Lynden-Bell in the AGN context 41, 42]. A magnetic tower is an axisymmetric magnetic configuration that arises when a system of nested closed flux surfaces, anchored in a differentially-rotating disk, is twisted and, as a result, inflates, but when this inflation is controlled by a surrounding external pressure. The basic physical mechanism of this process can be described as follows (see Fig. 5 ).

Consider a thin conducting disk with some vertical magnetic flux frozen into it. Let us assume that initially the magnetic field has a dipole-like topology (see Fig. [5), with the two footpoints of each field line located at different radii on the disk. Now let the disk rotate nonuniformly (e.g., a Keplerian disk). Then, each field line $\Psi$ is twisted at a rate $\Delta \Omega(\Psi)$ equal to the difference in angular velocities of its two footpoints. Correspondingly, toroidal magnetic flux is generated from the poloidal flux. The pressure of the toroidal field pushes the flux surfaces out, against the poloidal field tension. It is assumed that during the initial stages of this process the gas pressure, as well as the gravitational and inertial forces, are negligible in the magnetosphere, so the magnetic field is force-free. Then the expansion is uncollimated, typically at a $60^{\circ}$ angle with respect to the rotation axis, as shown in Figure 5b (e.g., Refs. 52, 53 ). However, as was shown by Lynden-Bell[41], if there is some, no matter how small, external gas pressure $P_{\text {ext }}$ surrounding the expanding magnetosphere, then the sideways expansion ceases once $B^{2} / 8 \pi$ drops down to $P_{\text {ext }}$. Subsequently, as again was shown by Lynden-Bell[41], the twisted magnetosphere continues to expand in the vertical direction (Fig. [5.) and eventually forms a cylindrical column that Lynden-Bell termed a magnetic tower (see Fig. 5 $\mathrm{d}$ ). If the external pressure outside of the tower is kept constant and uniform, then the top of the tower rises at a constant speed. Plasma inertia never plays any role; the entire evolution is a sequence of force-free magnetostatic equilibria with a pressure balance between the external gas outside of the tower and the magnetic field inside.

Note that the assumption that both ends of the field lines connect to the disk is not essential. A similar behavior is encountered in the case of a rotating conducting disk magnetically connected to a central star (e.g., Refs. [53, 54]) or a rotating black hole [55]. We believe that the model is also applicable to the millisecondmagnetar central engine scenario for GRBs.

To get a physical feeling of how the magnetic tower grows, it is instructive to derive some simple orderof-magnitude estimates and scaling relationships. The main input parameters that set the characteristic phys- 
ical scales are the total poloidal magnetic flux $\Psi_{0}$ (per unit toroidal angle) in the tower, the characteristic differential rotation rate $\Delta \Omega$, and the external pressure $P_{\text {ext }}$. Let us now ask how the main parameters of the tower, namely, its radius $R_{0}$, the typical magnetic field $B_{0}$, and the growth velocity $V_{\text {top }}$, scale with the three input parameters.

First, the radius of the tower, $R_{0}$, and the characteristic poloidal magnetic field, $B_{\mathrm{pol}}$ are related via

$$
B_{\mathrm{pol}} \sim B_{0} \equiv \frac{\Psi_{0}}{R_{0}^{2}} .
$$

The radius adjusts so that the magnetic pressure inside the tower equals $P_{\text {ext }}$. From the force-free balance inside the tower we expect $B_{\phi} \sim B_{\text {pol }} \sim B_{0}$; hence, the total magnetic field strength at the outer edge of the tower is also of order $B_{0}$. Then, from the the condition of pressure balance across the tower's wall we get

$$
B_{0} \sim \sqrt{8 \pi P_{\mathrm{ext}}},
$$

and so

$$
R_{0} \sim\left(\frac{\Psi_{0}^{2}}{8 \pi P_{\mathrm{ext}}}\right)^{\frac{1}{4}}
$$

To estimate the growth rate of the tower, note that the toroidal magnetic flux $\chi$ is continuously generated from the poloidal flux $\Psi_{0}$ by the differential rotation

$$
\chi=2 \pi \Psi_{0} N=\Psi_{0} \Delta \Omega t .
$$

Taking the tower to be a cylinder with radius $R_{0}$ and height $Z_{\text {top }}$, we get $B_{\phi} \sim \chi / R_{0} Z_{\text {top }}=$ $\left(\Psi_{0} / R_{0} Z_{\text {top }}\right) \Delta \Omega t=B_{0} \Delta \Omega t\left(R_{0} / Z_{\text {top }}\right)$. However, as stated earlier, the typical toroidal field in the tower should be of the order of $B_{0}$; therefore, the height of the tower increases steadily as

$$
Z_{\text {top }}(t) \sim R_{0} \Delta \Omega t .
$$

In other words, the tower grows at the speed of order of the typical differential rotation velocity $R_{0} \Delta \Omega$. If the external pressure does not change, the radius of the tower, determined by Eq. (12), stays constant during its growth; therefore, after many turns $(\Delta \Omega t \gg 1), Z_{\mathrm{top}} \gg R_{0}$, i.e., the tower becomes slender.

Since the first analytical solution proposed by LyndenBell[41], the magnetic tower concept is becoming more and more accepted by the astrophysical community. The formation and evolution of magnetic towers have been studied in numerical simulations $[56,57,58,59,60,61,62$ and even in real laboratory experiments 63,64$]$.

An interesting question is the flow of energy through a magnetic tower. As one can easily see, Poynting flux flows up from the disk along the inner segment of each field line and down to the disk along the outer segment. Indeed, for each field line $\Psi$, the inner, fasterrotating footpoint (1) performs work on the magnetic field, $W_{1} \sim I_{\text {pol }}[\Psi(1)] \Omega(1)$ (per unit time and unit poloidal flux). The corresponding decelerating torque per unit flux is $\tau_{1} \sim I_{\text {pol }}[\Psi(1)]$. In turn, the magnetic field exerts an accelerating torque per unit flux $\tau_{2} \sim I_{\mathrm{pol}}[\Psi(2)]$ on the outer disk footpoint of the same field line. Correspondingly, it performs work at a rate $W_{2} \sim I_{\mathrm{pol}}[\Psi(2)] \Omega(2)$. Because of force-free equilibrium in the tower, $I_{\mathrm{pol}}[\Psi(1)]=I_{\mathrm{pol}}[\Psi(2)]=I_{\mathrm{pol}}(\Psi)$, and so $\tau_{1}=\tau_{2}$, i.e., all the angular momentum extracted magnetically from point 1 is transferred to point 2 . The two energy flows, on the other hand, are not equal: since $\Omega(1)>\Omega(2)$, the energy extracted from point 1 along the inner segment of the field line is greater than the energy that flows down along the outer segment and is deposited in the disk at point 2 . The difference, proportional to $I_{\mathrm{pol}}(\Psi) \Delta \Omega(\Psi)$, is the power driving the expansion of the tower. A part of it goes into filling the growing volume of the tower with magnetic energy, and the rest goes into performing work against external gas pressure and driving the shock through the star.

It is interesting to note that the total vertical Poynting flux in the two segments only involves the differential rotation $\Delta \Omega=\Omega(1)-\Omega(2)$, but is independent of the absolute rotation itself. This is because we are dealing here with a force-free equilibrium, so that $I_{\mathrm{pol}}$ is constant along the entire length of a field line; in particular, it has the same sign on the two segments of the field line, and hence so does $B_{\phi}$. The situation is drastically different in the relativistic-rotation case where both fieldline segments extend beyond their respective light cylinders. In that case, one no longer has a force-free equilibrium along the entire field line; in particular, equilibrium breaks down at the farthermost tip of the line where the two segments join. As a result, the signs of $I_{\text {pol }}$ (and hence of $B_{\phi}$ ) on the two segments are opposite, which corresponds to both segments being swept back. Consequently, the Poynting flux is outward along both segments. A similar situation arises in the non-force-free MHD case; the two field-line segments are then swept back by plasma inertia if they extend beyond the Alfvén point. This again results in an outward Poynting flux along both segments. In both of these cases, the total vertical Poynting flux depends on the absolute rotation rates $\Omega(1)$ and $\Omega(2)$ themselves, as opposed to just their difference.

\section{Magnetic Tower Driving a Shock through a Star}

There are several reasons that make the magnetic tower an attractive model for the formation and propagation of a magnetically-dominated jet through a star within the collapsar model for GRBs (and core-collapse 
$\mathrm{SNe}$ ). First, a configuration where all the field lines close back onto the central engine (an accretion disk or a magnetar) is natural for a field created by a dynamo with zero net flux. In addition, mixing of baryons from the stellar envelope via Kelvin-Helmholtz and/or Rayleigh-Taylor instabilities may be inhibited by the magnetic field.

In order to apply the magnetic tower model to the collapsar scenario, we first want to make some modifications to Lynden-Bell's picture 15]. Specifically, we take into account the high-pressure cocoon that surrounds and confines the tower (see Fig. 6). In our model, the magnetic tower grows very rapidly and acts as a piston driving a shock ahead of itself. The shocked gas above the tower has very high pressure; it squirts sideways and forms backflows that fill the cocoon around the tower. Therefore, the external pressure confining the tower is no longer an arbitrary parameter, as in Lynden-Bell's model, but is determined by the jump conditions across the shock surrounding the cocoon and across the contact discontinuity between the cocoon and the tower. The external unperturbed pressure of the star is actually irrelevant; it should thus be excluded from our three input parameters. Instead, the expansion is controlled by the ram pressure related to the gas inertia; therefore, we replace $P_{\text {ext }}$ by the unperturbed stellar density $\rho_{0}$ in the list of basic dimensional parameters (along with $\Psi_{0}$ and $\Delta \Omega$ ) that determine the physical scales in our problem. This change is an important difference between our model and Lynden-Bell's.

The actual situation is complicated further by the twodimensional character of the problem. Since the sound speed in the cocoon is very high, gas pressure tends to be equalized throughout the cocoon. This expectation is supported by the hydrodynamic simulations of the collapsar model [50, 51], which show a relatively weak (just a factor of 5 or 10) variation of the cocoon pressure along its length. This is very moderate compared to the corresponding variations of the unperturbed stellar density and pressure, which both vary by many orders of magnitude along the vertical extent of the cocoon. Thus, the gas pressure is very high everywhere in the cocoon and so the cocoon also drives a sideways shock into the star. The boundary between the tower and the cocoon is a contact discontinuity, whereas the boundary between the cocoon and the rest of the star is a two-dimensional strong shock of some complicated shape.

\section{Simple Estimates}

Let us now show how the basic parameters of the growing magnetic tower scale with $\Psi_{0}, \Delta \Omega$, and $\rho_{0}$. We shall ignore any non-uniformity of the gas pressure in the cocoon. Also, for simplicity we shall use one-dimensional shock jump conditions. Since the pressure of the unperturbed stellar gas upstream of the shock is neglected, the shock is strong. Assuming an adiabatic index of $5 / 3$, the shock velocity with respect to the unperturbed gas is $V_{s}=4 / 3 V_{\text {top }}$, whereas the pressure in the post-shock region (i.e., in the cocoon) is expressed in terms of the velocity of the piston $V_{p} \equiv V_{\text {top }}$ and the upstream gas density $\rho_{0}$ as

$$
P_{\mathrm{top}}=\frac{3}{4} \rho_{0} V_{s}^{2}=\frac{4}{3} \rho_{0} V_{\mathrm{top}}^{2} .
$$

By comparing this with the pressure balance $P_{\text {top }} \simeq$ $B_{0}^{2} / 8 \pi$ at the contact discontinuity at the top of the tower, we see that the tower grows with a velocity of order the Alfvén speed corresponding to $\rho_{0}$ :

$$
V_{\mathrm{top}} \sim V_{A} \equiv \frac{B_{0}}{\sqrt{4 \pi \rho_{0}}}=\frac{\Psi_{0}}{R_{0}^{2} \sqrt{4 \pi \rho}} .
$$

But, as we have shown earlier, $V_{\text {top }}$ should be of the order of $R_{0} \Delta \Omega$. Thus, we obtain the scaling of $R_{0}$ with $\Psi_{0}, \Delta \Omega$, and $\rho_{0}$ :

$$
R_{0} \sim\left(\frac{\Psi_{0}}{\Delta \Omega}\right)^{1 / 3}\left(4 \pi \rho_{0}\right)^{-1 / 6}
$$

We can also relate the radius of the tower to the radius of the central rotating conductor. Estimating the poloidal flux as $\Psi_{0} \sim B_{d} R_{d}^{2}$, where $B_{d}$ and $R_{d}$ are the typical magnetic field and the radius of the the base of the tower (e.g., the inner part of the accretion disk), we we get $B_{0} \sim B_{d}\left(R_{d} / R_{0}\right)^{2}$ and hence

$$
\frac{R_{0}}{R_{d}} \sim\left(\frac{\tilde{V}_{\mathrm{A}, \mathrm{d}}}{V_{d}}\right)^{1 / 3} .
$$

Here $V_{d} \equiv R_{d} \Delta \Omega$ is the characteristic differential rotation velocity of the central conductor and

$$
\tilde{V}_{\mathrm{A}, \mathrm{d}} \equiv \frac{B_{d}}{\sqrt{4 \pi \rho_{0}}}
$$

is a composite Alfvén speed involving the disk magnetic field and the unperturbed star's plasma density; it doesn't have a direct physical meaning and thus can be arbitrarily high.

Notice that, as the tower makes its way through the star, $\Psi_{0}$ and $\Delta \Omega$ remain unchanged, whereas the third parameter, the unperturbed density $\rho_{0}$ at the top of the tower, changes. It drops rather rapidly for a typical collapsar progenitor and so the radius of the tower increases as the it grows. Thus we expect that, in a realistic situation, the tower will not be a straight cylinder, as we have assumed here; instead, its radius will be some function of the vertical coordinate $z$. However, as is seen from Eq. (17), $R_{0}$ scales only weakly with $\rho_{0}\left(\right.$ as $\left.\rho_{0}^{-1 / 6}\right)$, which somewhat justifies the constant-radius approximation. 


\section{The Numbers}

Now let us make some quantitative estimates based on the above scaling relationships; unavoidably, these estimates will be very crude. For definiteness, consider the accreting black hole scenario for the central engine. We assume that the core of the star has collapsed into a black hole of fiducial mass $M=3 M_{\odot}$ with a gravitational radius $R_{g} \equiv G M / c^{2} \simeq 5 \mathrm{~km}$, and that some of the continuously infalling material has formed an accretion disk around the black hole. We take a fiducial disk radius of $R_{d} \simeq 6 R_{g} \simeq 3 \cdot 10^{6} \mathrm{~cm}$ and an initial poloidal flux (per unit toroidal angle) of $\Psi_{0}=$ $R_{d}^{2} B_{0} \simeq 10^{28} B_{15} R_{d, 6.5}^{2}$ in cgs units. This poloidal flux is being continuously twisted by the differential rotation of the disk, with characteristic angular velocity $\Delta \Omega=\Omega_{K}\left(R_{d}\right) \simeq 4 \cdot 10^{3} \sec ^{-1}\left(M / 3 M_{\odot}\right)^{1 / 2} R_{d, 6.5}^{-3 / 2}$.

Taking the fiducial stellar background density to be $\rho_{0}=10^{6} \mathrm{~g} / \mathrm{cm}^{3}$, the tower outer radius can be estimated as

$$
R_{0} \sim R_{d}\left(\frac{\tilde{V}_{A, d}}{V_{d}}\right)^{\frac{1}{3}}=3 R_{d}\left(\frac{B_{d, 15}}{R_{d, 6.5} \Delta \Omega_{3.5} \sqrt{\rho_{0,6}}}\right)^{1 / 3},
$$

resulting in $R_{0} \simeq 10^{7} \mathrm{~cm}$ for our fiducial parameter values. Then we get the following expressions for all the other parameters:

$$
\begin{aligned}
B_{0} & \equiv \frac{\Psi_{0}}{R_{0}^{2}}=B_{\mathrm{d}}\left(\frac{R_{\mathrm{d}}}{R_{0}}\right)^{2} \\
& \simeq 0.1 B_{\mathrm{d}}\left(\frac{R_{d, 6.5} \Delta \Omega_{3.5}}{B_{\mathrm{d}, 15}}\right)^{2 / 3} \rho_{0,6}^{1 / 3} \simeq 10^{14} \mathrm{G} ;
\end{aligned}
$$

$\left.V_{A, 0} \equiv \frac{B_{0}}{\sqrt{4 \pi \rho_{0}}}=3 \cdot 10^{10} \mathrm{~cm} / \sec B_{d, 15}^{1 / 3} R_{d, 6.5}^{2 / 3} \Delta \Omega_{3.5}^{2 / 3} \rho_{0,62}^{-12} 2\right)$

Notice that our crude estimate results in $V_{\text {top }} \sim V_{A, 0}$ being comparable to the speed of light $c$. Therefore, a fully-relativistic treatment of the problem would be more appropriate (see Sec. „for discussion). Such a treatment, however, lies beyond the scope of the current paper.

Also, we can estimate the post-shock pressure in the hot cocoon above the tower as

$$
P_{\text {top }} \simeq \frac{B_{0}^{2}}{8 \pi} \simeq 4 \cdot 10^{26} \mathrm{erg} \mathrm{cm}^{-3} B_{0,14}^{2} .
$$

where $B_{0,14} \equiv B_{0} /\left(10^{14} \mathrm{G}\right)$. At such high energy density the radiation pressure probably dominates over the gas pressure; we can therefore estimate the plasma temperature in the post-shock region as

$$
T_{\text {top }} \simeq\left(\frac{3 P_{\text {top }}}{a}\right)^{1 / 4} \simeq 2 \cdot 10^{10} \mathrm{~K} \simeq 2 \mathrm{MeV},
$$

where $a \simeq 7.6 \cdot 10^{-15} \mathrm{erg} \mathrm{cm}^{-3} \mathrm{~K}^{-4}$. On the other hand, since we are dealing with a strong hydrodynamic shock between the cocoon and the unperturbed stellar material, the baryon density in the cocoon is simply $4 \rho_{0}$, and so the baryon rest-mass energy density is $4 \rho_{0} c^{2} \simeq 4 \cdot 10^{27} \mathrm{erg} \mathrm{cm}^{-3} \rho_{0,6}$, and hence still exceeds the radiation/pair energy density by an order of magnitude. The total magnetic energy contained in the tower of height $Z_{\text {top }}$ can be estimated as

$$
E_{\mathrm{mag}}(t) \simeq 2 \pi R_{0}^{2} Z_{\mathrm{top}}(t) \frac{B_{0}^{2}}{8 \pi} \simeq 2 \cdot 10^{50} \operatorname{erg} R_{0,7}^{2} Z_{\mathrm{top}, 9} B_{0,14}^{2},
$$

which is a noticeable fraction of a typical GRB energy.

\section{DISCUSSION}

\section{Transition to Relativistic Expansion Regime and the Jet Opening Angle}

As we have shown above, the tower radius $R_{0}$, and hence its growth velocity $V_{\text {top }} \sim \Delta \Omega R_{0}$ scale with background density as $\rho_{0}^{-1 / 6}$. Therefore, as the tower expands into the outer regions of the star, $V_{\text {top }}$ inevitably reaches the speed of light at some critical density $\rho_{0 \text {,rel }}$. For our fiducial values $B_{d}=10^{15} \mathrm{G}, R_{d}=3 \cdot 10^{6} \mathrm{~cm}$, and $\Delta \Omega=3 \cdot 10^{3} \mathrm{sec}^{-1}$, this critical density for the transition to the relativistic regime is $\rho_{0, \text { rel }} \sim 10^{6} \mathrm{~g} / \mathrm{cm}^{3}$ [see eq. (22)]. For a typical massive stellar GRB progenitor this corresponds to a distance $Z_{\text {rel }}$ from the center on the order of $10^{8} \mathrm{~cm}$; according to Eq. (20), the corresponding radius of the tower is $R_{\text {rel }} \sim 10^{7} \mathrm{~cm}$.

Our non-relativistic model becomes becomes invalid at this point and the subsequent expansion of the tower calls for a relativistic generalization of the magnetic tower model, which is beyond the scope of this paper. However, we probably can derive some physical insight into the relativistic regime by looking at the results of fullyrelativistic hydrodynamic simulations [51]. In those simulations the relativistic jet remained collimated as it propagated through the star, across several orders of magnitude in $\rho_{0}$. This has been attributed to recollimation shocks in the cocoon and to relativistic beaming in the jet. But the physical processes in the cocoon should not change if we replace the inner relativistic hydrodynamic jet with a relativistic magnetic tower. Furthermore, we expect some additional magnetic collimation due to the hoop stress. To sum up, we expect the magnetic tower to remain collimated even after it transitions into the relativistic regime. In particular, we suggest that the final opening angle of the outflow will be about the inverse aspect ratio of the tower at relativistic transition:

$$
\Delta \theta \lesssim \frac{R_{0, \text { rel }}}{Z_{\text {rel }}} \simeq 0.1 .
$$

Whether this prediction is true will have to be determined by a fully-relativistic analysis and by relativistic 
MHD simulations, which we hope will be completed in the near future.

\section{Small-Scale Structure of the Outflow}

The physical picture presented in this paper, with its smooth coherent magnetic structure, is just an idealization used to get the main ideas across in the clearest way possible. The actual magnetic field, especially if it is produced by a turbulent dynamo in the PNS or in an accretion disk, will of course be different from such a simple axisymmetric system of nested flux surfaces. Instead, it may consist of an ensemble of loops of different sizes and orientations. It may thus have a highly-intermittent substructure on smaller scales, both temporal and spatial. However, each of these smaller magnetic structures is subject to the same physical processes as the simple large-scale configuration: twisting by differential rotation and a subsequent inflation controlled by the external pressure of the cocoon and of the other loops growing at the same time. As a result, a more realistic picture may look like a train of spheromak-like plasmoids, pushing each other out along the axis (see Fig. 7). Hoop stress still works inside each of them, and so the overall dynamical effect may be qualitatively similar to that of a single tower. A similar picture may also develop if a large-scale twisted magnetosphere becomes unstable to the kink instability and undergoes flux conversion as a result, breaking up into smaller plasmoids (see below). In either case, the resulting multi-component structure of the outflow may be responsible for the observed intermittency in GRBs.

\section{Effect of MHD Instabilities on the Tower Evolution}

One of greatest uncertainties in our model is the effect of MHD instabilities in the highly twisted magnetic structure. In this section we discuss two such instabilities: Rayleigh-Taylor and kink.

(i) Rayleigh-Taylor (or its magnetic counter-part, Kruskal-Schwarzschild) instability may occur at the interface between the growing magnetic bubble and the overlying colder, denser stellar material. This instability is expected to affect purely hydrodynamic fireball models as well; if anything, strong magnetic fields are expected to suppress it somewhat, although probably not completely. The Rayleigh-Taylor instability may cause splitting of a coherent magnetic structure into several separate strands interlaced with stellar matter [30, 40]. For example, in the Arons model[40], the stellar envelope is "shredded" by the nonlinear Rayleigh-Taylor "fingers". This leads to creation of several evacuated channels that allow the electromagnetic energy produced near the central engine to escape through the star. Arons further argued that these channels suffer only a small amount of mixing with the non-relativistic stellar material due to the Kelvin-Helmholtz instability. In light of his work, we think that our magnetic cavity and/or the subsequentlyformed magnetic towers may also suffer from fragmentation into Rayleigh-Taylor "fingers". However, as is well known, initially small-scale fingers quickly merge to form a few large ones in the nonlinear stage. Therefore, one does not expect strong mixing of the baryons from the stellar envelope into the magnetosphere. The exact geometry of the outflow may be affected somewhat and a strong time-variability may develop, but, overall, we expect the outflow to survive. More research is needed in order to assess the implications of this instability for our scenario.

(ii) As the magnetic configuration is twisted up, it may become prone to a non-axisymmetric kink-like instability. This may happen both during the pulsar-in-a-cavity phase and during a later magnetic tower phase. Whereas the stability of the pulsar-in-a-cavity has not yet been studied, several non-relativistic 3D MHD simulations have recently addressed 58, 61, 62] the kink instability of magnetic towers (although not in the GRB context). They seem to indicate that during the first few rotation periods, the tower is stabilized by the surrounding highpressure gas, but at later times a large-scale external kink does develop. As a result, the tower's general shape becomes helical. This, however, does not immediately lead to the overall disruption of the tower; even though the configuration is nonaxisymmetric, its main morphological features remain similar to those in the axisymmeitrc case 61. Similar conclusions have been reached by Nakamura \& Meier 65] in their 3D-MHD study of Poyntingflux-dominated jets propagating through a stratified external medium. In particular, these authors found that a steep external pressure gradient forestalls the instability onset. When the instability does eventually develop, the resulting helical structures saturate and do not develop into full MHD turbulence. Important theoretical evidence supporting the idea of the external pressure stabilization follows from Königl \& Choudhuri's analysis of a force-free magnetized jet confined by an external pressure 66. They showed that a non-axisymmetric helical equilibrium state becomes energetically favorable (conserving the total magnetic helicity in the jet) only when the pressure drops below a certain critical value. If this happens and the external kink does go unstable, then this non-axisymmetric equilibrium can be interpreted as the end point of the non-linear development of the instability.

In addition to the above non-relativistic studies, several first steps have recently been undertaken to understand the stability of relativistic jets, in particular in the framework of relativistic force-free electrodynamics 67 , 68]. However, so far as we know, there have been no formal stability studies of relativistic magnetic towers 
to date. Such studies, both analytical and numerical, are clearly needed. They would have to take into account several stabilizing effects. First, as Tomimatsu et al. have found in their linear stability analysis of a narrow rotating relativistic force-free jet, rapid field-line rotation inhibits the kink instability [68]. Second, we expect that the tower expansion should quickly transition to the relativistic regime (see Sec. ${ }_{\text {n) }}$ ), eventually reaching a very large $\gamma$-factor. Once this happens, the relativistic time delay may effectively stabilize the tower [17]. This is because MHD instabilities grow on the local Alfvéncrossing time in the fluid frame and hence much slower in the laboratory frame. As a result, even if instabilities are excited, they do not have enough time to develop before the break-out of the flow from the star.

If it does develop, the kink is probably the most dangerous instability and may lead to a significant, although perhaps temporary, disruption. Such a disruption, however, is not necessarily a bad thing: the tower may be able to reform after being disrupted (as is seen in laboratory experiments 64]) and the resulting non-steady evolution may provide a plausible mechanism for rapid variability seen in gamma-ray bursts. Also, as a result of such disruption, a significant fraction of the toroidal magnetic field energy may be dissipated into thermal energy [69, 70], which may in fact contribute to the acceleration of the Poynting-flux dominated outflow and to powering the prompt gamma-ray emission at later times [10, 16, 17, 18]. In any case, the kink, and especially its nonlinear outcome, is a serious issue that needs to be addresses in the future. Axisymmetric sausage instability also needs to be investigated.

An important aspect of our problem is that we are actually interested not so much in the instability onset or its early linear development, but rather in the longterm (many rotation periods) nonlinear evolution and its overall effect on the outflow. An important consideration that then needs to be taken into account is the conservation of magnetic helicity. Differential rotation leads to a continuous injection of helicity into the system (of opposite signs in the two hemispheres). In its nonlinear stage, the kink instability may lead to conversion of some of the toroidal magnetic flux to poloidal flux (in our geometry); however, it will not destroy the magnetic helicity accumulated in the cavity. Thus, whatever the resulting configuration will be, it will have to be consistent with a growing amount of helicity. If some of the new poloidal flux becomes detached from the base due to reconnection (which may actually be strongly inhibited, see discussion in Section ${ }_{a}$ ), then the resulting configuration may resemble a train of plasmoids (Fig. 17), similar to Section may in fact imagine a cyclic process involving the growth of the tower for several rotation periods, followed by flux conversion due to the kink, followed by reconnection and subsequent ejection of a plasmoid carrying the magnetic helicity (and some of the magnetic energy) injected dur- ing the given cycle.

It is also interesting to make the following observation. An unbounded relativistic force-free outlow from a rotating conductor is expected to be stable. On the other hand, in the case of a confined closed magnetosphere with field lines subject to differential rotation, such as our pulsar-in-a-cavity problem or a magnetic tower, kink is expected to develop. At the same time, as we discussed in Sec. $₫$ the outflow is uncollimated in the first case but is collimated in the second case. This suggests that there may be a deep connection between stability and lack of collimation of axisymmetric relativistic force-free flows.

Finally, we would like to reiterate that a proper treatment of these problems requires a time-dependent relativistic force-free or full (preferably relativistic) MHD analysis and simulations (see Sec. ${ }_{\mathrm{n}}$ ).

\section{Reconnection}

Another important issue is magnetic reconnection across the equatorial current sheet in the pulsar-in-acavity magnetosphere or across the separatrix current sheet in the magnetic tower. This process may, in principle, lead to the break-up of a single structure into several smaller spheromak-like plasmoids (see Fig. 7), similar to the cyclic bevavior (involving reconnection) suggested for magnetospheres of accreting young stars 71,72 . The expected size of the plasmoids and their production rate are presently not known.

We would like to remark, however, that fast a reconnection is difficult to achieve deep inside a collapsing star [15]. The reason for this is that fast Petschek-like reconnection is now believed to be possible only in collisionless environments, such as the Solar corona, Earth magnetosphere, and tokamak plasmas. The plasma inside a collapsing star, on the other hand, is highly collisional [15], so that classical collisional resistivity dominates over all other non-ideal effects in generalized Ohm's law. It is now believed, on the basis of numerical simulations [73, 74], theoretical analysis 75, 76], and laboratory experiments 77], that reconnection in such a situation proceeds in the very slow Sweet-Parker 78, 79] regime, whereas Petschek's [80] fast mechanism fails. It is of course not obvious that this conclusion can be extended to the highly relativistic and optically-thick electron-positron plasma in the deep interior of a collapsing star. However, at the very least, this observation casts a serious doubt on the possibility of fast and efficient large-scale reconnection in this environment. As the magnetic tower grows and eventually breaks out of the star, however, the plasma cools and the particle density in it drops rapidly. At some point, the plasma becomes collisionless (from the reconnection point of view) and this opens up the possibility of reconnection and the corresponding delayed magnetic 
energy release. Indeed, post-breakout reconnection in relativistic Poynting-flux-dominated outflows has been invoked as a plausible mechanism for powering GRB emission 10, 11, 13, 16, 17, 17, 81].

\section{Nickel Production}

A key issue for long-duration GRBs is the required production of ${ }^{56} \mathrm{Ni}$. The supernovae observed to accompany these GRBs (SN-GRBs) belong to Type Ibc [82, 83]. They are believed to require radioactive ${ }^{56} \mathrm{Ni}$ to heat the ejecta after the initial expansion of the star. The brightest SN-GRBs (e.g., SN1998bw and SN2003dh) require up to several $0.1 \mathrm{M}_{\odot}$ of ${ }^{56} \mathrm{Ni}$, as inferred from peak optical brightness, although on average SN-GRBs do not need more ${ }^{56} \mathrm{Ni}$ than the local population of $\mathrm{SNe} 82$. In fact, some SN-GRBs (e.g., GRB060505 and GRB060614; see Refs. [84, 85, 86]) may produce little or no ${ }^{56} \mathrm{Ni}$ [87].

In models of core-collapse $\mathrm{SNe},{ }^{56} \mathrm{Ni}$ is produced in material heated to $T \gtrsim T_{\mathrm{Ni}} \sim 5 \times 10^{9} \mathrm{~K}$ by the explosion shock launched in the core of the star. The amount of ${ }^{56} \mathrm{Ni}$ produced depends on the mass inside of the expanding shock when its temperature declines below $T_{\mathrm{Ni}}$. This happens when its radius has reached

$$
R_{\mathrm{Ni}} \sim\left(\frac{3 E}{4 \pi a T_{\mathrm{Ni}}^{4}}\right)^{1 / 3} \sim 4 \times 10^{8} E_{51} \mathrm{~cm}
$$

where $E=E_{51} \times 10^{51}$ erg is the explosion energy and $a$ is the radiation constant. The mass inside $R_{\mathrm{Ni}}$ depends on the progenitor structure and on the expansion or contraction that took place before the shock reached a given mass element. In particular, little ${ }^{56} \mathrm{Ni}$ is produced by a shock, even if very powerful, if it is launched into a low density material, as it may occur if a weak initial explosion pre-inflates the stellar core so that little mass remains within a few $10^{8} \mathrm{~cm}$ when the subsequent strong shock arrives. Production of $\sim 0.1 M_{\odot}$ of ${ }^{56} \mathrm{Ni}$ usually requires $\sim 10^{51}$ ergs to be deposited isotropically by a quasi-spherical shock within $\sim 1$ sec, so that little preexpansion of the star occurs. Brightest supernovae, e.g., SN1998bw, require energies of up to $\sim 10^{52}$ ergs to make the $\sim 0.5 M_{\odot}$ inferred from lightcurve modeling.

The requirement of fast $(\lesssim 1 \mathrm{sec})$, isotropic deposition of energy for production of ${ }^{56} \mathrm{Ni}$ is a serious challenge for models of the SN-GRB central engine, because the GRB engine must last for $\gtrsim 10 \mathrm{sec}$ for relativistic ejecta to escape the star and because GRBs are believed to be collimated explosions. The high degree of beaming and long timescale for energy deposition makes collapsar jets incapable of producing anywhere near the required amounts of ${ }^{56} \mathrm{Ni}[3]$. Therefore, in the original collapsar model, with a black hole accretion disk as the central engine, the ${ }^{56} \mathrm{Ni}$ is produced by a slower bi-conical disk wind that constitutes a distinct explosion component [3, 87].
In our magnetar model, on the other hand, ${ }^{56} \mathrm{Ni}$ can be produced behind a roughly spherical shock driven by the initial quasi-isotropic expansion of the magnetosphere. The expansion becomes collimated and a magnetic tower forms only at a later stage. Thus, the collimation process involves both a quick isotropic expansion followed by a beamed component. We feel that this modification to the magnetar scenario, i.e., the inclusion of the magnetosphere interaction with the surrounding star, strengthens its viability as a model for the long GRB central engine.

On the other hand, one cannot exclude the possibility that, if most of the energy produced by the central engine escapes through the narrow jet channel, giving rise to a GRB, then there may not be much energy left to explode the rest of the star. If this happens, then, eventually, almost all of the stellar material falls into a black hole. This would include all the ${ }^{56} \mathrm{Ni}$ that might have been produced, leaving no observable supernova signature (e.g., GRB060614).

\section{Pulsar Kicks}

Since most of the extracted rotational energy of the neutron star travels vertically through the two oppositely directed channels, a significant amount of linear momentum is also transported up and down and hence a reactive force is exerted on the NS from both sides Even a small imbalance in the reactive magnetic force may impact a sizable overall momentum to the NS. For example, taking the total initial rotational energy of the NS to be $E_{\text {rot }}=5 \cdot 10^{52} \mathrm{erg}$, the momentum transported out in each direction is $P=E_{\text {rot }} / 2 c \sim 10^{42} \mathrm{cgs}$ (it will be even larger if the propagation speed is less than $c$ ). Thus, just a $10 \%$ imbalance may result in the NS terminal velocity of order of $v_{\text {term }} \simeq 0.1 P / M_{\mathrm{NS}} \sim 300 \mathrm{~km} / \mathrm{sec}$.

\section{Suggestions for Numerical Simulations}

In this section we discuss a sequence of numerical studies of the interaction between a magnetar's magnetosphere and its birth environment, employing a range of plasma descriptions. Each of them will be able to address a subset of key issues with increasing degree of realism. For example, non-relativistic axisymmetric MHD simulations can address the following questions: What basic magnetic configuration results when the conditions we describe are set up? What is the overall magnetic field structure? When do magnetic towers form? How does the tower shape change as it expands into lowerdensity regions? How strongly is the magnetic field concentrated towards the axis? How does the Poynting flux depend on radius and height? What is the effect of the neutrino- or magneto-centrifugally-driven winds? To what degree does the cocoon help collimate and stabi- 
lize the tower? How rapidly do the cocoon walls spread laterally? Can the cocoon expansion result in the disruption of the star? In addition, some of the physical processes described in this paper may be relevant to other astrophysical systems, including non-relativistic central objects (e.g., planetary nebulae 48, 88]). For this reason, non-relativistic MHD simulations are of interest in themselves, as well as a first step toward fully-relativistic MHD. For example, recent non-relativistic MHD simulations indicate that the magnetic-tower mechanism can operate successfully in a variety of astrophysical environments 48, 57, 58, 60, 89], including collapsing massive stars [39].

Eventually, however, one will have to consider relativistic effects outside of the magnetar light cylinder or in the inner part of the black hole's accretion disk. This can be investigated using the relativistic force-free degenerate electrodynamics (FFDE) approach, valid in the case of a highly magnetized plasma with negligible pressure and inertia. Time-dependent force-free codes have recently been successfully used to study pulsar magnetospheres 90, 91, 92]. In the pulsar-in-a-cavity context, the cavity wall may be represented by a rigid conducting outer boundary. While this case may not be directly relevant to the realistic physical environment, some basic aspects of a bounded rotating magnetosphere may be understood using this description. Furthermore, the full magnetar-in-a-star problem can be investigated by a hybrid simulation employing a relativistic force-free code inside the cavity and a relativistic hydrodynamic simulation outside.

Finally, relativistic-MHD simulations will be able to address questions fundamental to the application of magnetar-driven magnetic towers to GRBs, including the beaming angle and angular distribution of energy flux and the growth of instabilities in the relativistic outflow. There now exist several relativistic MHD codes [36, 93, 94, 95, 96, 97, 98, 99] that can be used for this problem. Of interest would be a set of simulations with a range of plasma $\beta$. The low- $\beta$ simulations should match on to the FFDE case, at least qualitatively. Once these simulations are analyzed and the basic physical processes elucidated, $\beta$ can be gradually increased enabling an understanding of how plasma inertia and pressure affect the dynamics of the magnetosphere expansion and collimation.

The basic scenario described in this paper can initially be explored with axisymmetric simulations. However, in order to assess the role of non-axisymmetric instabilities, such as the Rayleigh-Taylor, Kelvin-Helmholtz, and

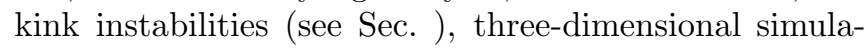
tions will eventually be necessary. One of the goals of such an investigation will be to estimate and the degree of mixing of the envelope baryonic material into the magnetosphere. In addition, one also would like to study the development and interaction of the MRI and the Parker instability.

The operation of the Parker instability, leading to the development of a highly-magnetized low-density corona, may be strongly influenced by neutrino cooling. Thus, a realistic treatment of the neutrino heating and cooling processes (as it has been done, e.g., by Burrows et al.[39]) is an essential physical ingredient of the overall problem.

In summary, numerical simulations of the full problem, including a detailed description of the central engine with relevant microphysical processes and neutrino transport, are desirable for a comprehensive understanding of the formation and evolution of a millisecond-magnetardriven magnetic tower inside a collapsing star. We believe that such simulations will very soon become technically feasible.

\section{CONCLUSIONS}

The core collapse of a massive rotating star may result in two distinct outcomes, both plausible candidates for the GRB central engine. The first one is a stellar-mass black hole with an accretion disk. The second is a neutron star. In this second scenario, the young neutron star formed as a result of core collapse has to be a millisecond magnetar in order to be relevant for GRBs.

In this paper we mostly focus on the millisecondmagnetar scenario, although many features of our model are also applicable to the black-hole case, which we have considered in our previous paper (Ref. 15]). Of particular interest to us is the interaction between the rapidlyrotating magnetar's magnetosphere and the surrounding infalling stellar envelope. We argue that the stellar material provides a confining (ram) pressure that has a strong effect on both the size and the shape of the magnetosphere. Namely, it can channel the highly-magnetized outflow originating from the proto-neutron star into two collimated magnetic towers.

More specifically, we suggest that the stalled bounce shock - a common feature in models of core-collapse supernovae - plays a role of a cavity that confines the magnetosphere. The cavity's radius, determined by the balance between the pressure of the hot neutrino-heated gas and the ram pressure of the infalling material, stays quasi-stationary at $R_{0} \simeq 200 \mathrm{~km}$ during the first few hundreds of milliseconds after the bounce. To get a qualitative physical feeling for what happens to the magnetar magnetosphere during this stage, we introduce an idealized fundamental-physics problem that we call the Pulsar-in-a-Cavity problem[32]. A large part of our paper (Sec. . $)$ is devoted to investigating this problem. For simplicity, we consider it under the force-free assumption. We show that if the radius of the cavity is larger than the pulsar light-cylinder radius, the magnetic field inside the cavity continuously winds up. Then, the toroidal field strength and hence the magnetic spin-down luminosity of 
the pulsar increase, roughly linearly with time. The magnetic energy in the cavity grows quadratically with time. We then estimate that in the context of a millisecond magnetar inside a collapsing star the magnetic field becomes dynamically important after a few hundred turns. This leads to a subsequent revival of the stalled shock and may result in a successful magnetically-driven explosion. As long as the expansion of the cavity is non-relativistic, the toroidal magnetic field inside it remains larger than the poloidal magnetic and electric fields. As a result, the hoop-stress collimates the Poynting-flux-dominated outflow into two oppositely-directed vertical channels.

We suggest that these magnetic outflows (either in the millisecond-magnetar or the accreting black hole scenario) should be described in terms of the magnetic tower concept, introduced by Lynden-Bell in the AGN context 41]. Correspondingly, we investigate the propagation of a magnetic tower inside a star (see also Ref. [15]). In our model, we modify Lynden-Bell's picture by considering that the tower expansion is supersonic with respect to the unperturbed stellar gas. We envision the growing magnetic tower acting as a piston that drives a strong shock through the star. The hot shocked stellar material between the shock and the tower forms a high-pressure cocoon that envelopes the tower and provides the collimating pressure. In other words, the tower in our model is confined not by the pressure of the background stellar material, but by its inertia; the strong shock and the cocoon act as mediators that convert the inertial support into the pressure support ultimately acting on the tower. The entire configuration grows vertically with time and eventually reaches the star's surface, thereby providing a narrow baryon-clean channel for the Poynting-flux dominated jet, surrounded by a less-collimated hot cocoon outflow.

Finally, we discuss the astrophysical implications of our model for GRBs and core-collapse supernovae, such as ${ }^{56} \mathrm{Ni}$ production and pulsar kicks. In addition, we discuss the role of MHD instabilities, most notably, the kink, in our scenario. We also assess the prospects for magnetic reconnection and find that it should be strongly inhibited in the central parts of the collapsing star owing to the high plasma collisionality there. Finally, we outline a set of numerical studies that we believe need to be done.

We would like to thank Profs. Jerry Ostriker and Russell Kulsrud for stimulating discussions and encouragement. We are also grateful to J. Arons, A. Beloborodov, E. Blackman, P. Goldreich, J. Goodman, S. Komissarov, A. Königl, H. Li, M. Lyutikov, J. McKinney, C. Thompson, T. Thompson, A. Spitkovsky, H. Spruit, J. C. Wheeler, and E. Zweibel for useful comments and suggestions.

DAU's research has been supported by the National Science Foundation under Grant PHY-0215581 (PFC: Center for Magnetic Self-Organization in Laboratory and Astrophysical Plasmas). AIM acknowledges support from the Keck Fellowship at the Institute for Advanced Study. 
* Electronic address: uzdensky@astro.princeton.edu

$\dagger$ Electronic address: aim@ias.edu

[1] S. E. Woosley, Astrophys. J., 405, 273 (1993)

[2] B. Paczynski, Astrophys. J., 494, L45 (1998)

[3] A. I. MacFadyen \& S. E. Woosley, Astrophys. J., 524, $262(1999)$

[4] D. Proga,, A. I. MacFadyen, P. J. Armitage, \& M. C. Begelman, Astrophys. J., 599, L5 (2003)

[5] C. Thompson, Mon. Not. R. Astron. Soc., 270, 480 (1994)

[6] P. Meszaros \& M. J. Rees, Astrophys. J., 482, L29 (1997)

[7] H. K. Lee, R. A. M. J. Wijers, \& G. E. Brown, Phys. Reports, 325, 83 (2000)

[8] N. Vlahakis, \& A. Königl, Astrophys. J., 563, L129 (2001)

[9] M. H. P. M. van Putten \& E. C. Ostriker, Astrophys. J., 552, L31 (2001)

[10] G. Drenkhahn, \& H. Spruit, Astron. \& Astrophys., 391, 1141 (2002)

[11] M. Lyutikov \& R. Blandford, "Electromagnetic explosions in Gamma-Ray Bursts", Bull. Amer. Astron. Soc., 35, 622 (2003); e-print (astro-ph/0312347) (2003)

[12] M. H. P. M. van Putten \& A. Levinson, Astrophys. J., 584, 937 (2003)

[13] M. Lyutikov, New J. Phys., 8, 119 (2006)

[14] J. C. McKinney, Astrophys. J., 630, L5 (2005)

[15] D. A. Uzdensky \& A. I. MacFadyen, Astrophys. J., 647, $1192(2006)$

[16] D. Giannios \& H. Spruit, Astron. \& Astrophys., 430, 1 (2005)

[17] D. Giannios \& H. Spruit, Astron. \& Astrophys., 450, 887 (2006)

[18] D. Giannios, \& H. Spruit, "Spectral and timing properties of a dissipative GRB photosphere", submitted to Astron. \& Astrophys. (2007); e-print (astro-ph/0611385)

[19] S. Akiyama, J. C. Wheeler, D. L. Meier, \& I. Lichtenstadt, Astrophys. J., 584, 954 (2003)

[20] N. V. Ardeljan, G. S. Bisnovatyi-Kogan, \& S. G. Moiseenko, Mon. Not. R. Astron. Soc., 359, 333 (2005)

[21] E. G. Blackman, J. T. Nordhaus, \& J. H. Thomas, New Astron., 11, 452 (2006)

[22] R. C. Duncan, \& C. Thompson, Astrophys. J., 392, L9 (1992)

[23] C. Thompson \& R. C. Duncan, Astrophys. J., 408, 194 (1993)

[24] J. P. Ostriker \& J. E. Gunn, Astrophys. J., 164, L95 (1971)

[25] V. V. Usov, Nature, 357, 472 (1992)

[26] I. Yi \& E. G. Blackman, Astrophys. J., 494, L163 (1998)

[27] T. Nakamura, Progress of Theoretical Physics, 100, 921 (1998)

[28] H. Spruit, Astron. \& Astrophys., 341, L1 (1999)

[29] M. A. Ruderman, L. Tao, \& W. Kluzniak, Astrophys. J., 542, 243 (2000)

[30] J. C. Wheeler, I. Yi, P. Höflich, \& L. Wang, Astrophys. J., 537, 810 (2000)

[31] J. C. Wheeler, D. L. Meier, \& J. R. Wilson, Astrophys. J., 568, 807 (2002)

[32] D. A. Uzdensky \& A. I. MacFadyen, "Magnetar-Driven Magnetic Tower as a Model for Gamma-Ray Bursts and Asymmetric Supernovae", submitted to Astrophys. J.
(2006); e-print (astro-ph/0609047)

[33] T. A. Thompson, P. Chang, \& E. Quataert, Astrophys. J., 611, 380 (2004)

[34] LeBlanc, J. M. \& Wilson, J. R., Astrophys. J., 161, 541 (1970)

[35] D. L. Meier, R. I. Epstein, W. D. Arnett, \& D. N. Schramm, Astrophys. J., 204, 869 (1976)

[36] M. Shibata, Y. T. Liu, S. L. Shapiro, \& B. C. Stephens, Phys. Rev. D, 74, 104026 (2006)

[37] N. Bucciantini, T. A. Thompson, J. Arons, E. Quataert, \& L. Del Zanna, Mon. Not. R. Astron. Soc., 368, 1717 (2006)

[38] B. D. Metzger, T. A. Thompson, \& E. Quataert, "Proto-Neutron Star Winds with Magnetic Fields and Rotation", accepted to Astrophys. J. (2007); e-print (astro-ph/0608682)

[39] A. Burrows, L. Dessart, E. Livne, C. D. Ott, \& J. Murphy, "Simulations of Magnetically-Driven Supernova and Hypernova Explosions in the Context of Rapid Rotation", submitted to the Astrophys. J. (2007); e-print (astro-ph/0702539) (2007)

[40] J. Arons, Astrophys. J., 589, 871 (2003)

[41] D. Lynden-Bell, Mon. Not. R. Astron. Soc., 279, 389 (1996)

[42] D. Lynden-Bell, Mon. Not. R. Astron. Soc., 341, 1360 (2003)

[43] S. E. Woosley \& T. A. Weaver, Annual Rev. Astron. \& Astrophys., 24, 205 (1986)

[44] H. A. Bethe, Rev. Mod. Phys., 62, 801 (1990)

[45] P. Goldreich \& W. H. Julian, Astrophys. J., 157, 869 (1969)

[46] N. S. Kardashev, Sov. Astron., 14, 375 (1970)

[47] A. F. Illarionov \& R. A. Sunyaev, Astron. \& Astrophys., 39, 185. (1975)

[48] S. Matt, A. Frank, \& E. G. Blackman, Astrophys. J., 647, L45 (2006)

[49] F. C. Michel, Astrophys. J., 180, L133 (1973)

[50] A. I. MacFadyen, S. E. Woosley, \& A. Heger, Astrophys. J., 550, 410 (2001)

[51] W. Zhang, S. E. Woosley, \& A. I. MacFadyen, Astrophys. J., 586, 356 (2003)

[52] D. Lynden-Bell \& C. Boily, Mon. Not. R. Astron. Soc., 267, 146 (1994)

[53] D. A. Uzdensky, A. Königl, \& C. Litwin, Astrophys. J., 565, $1191(2002)$

[54] R. V. E. Lovelace, M. M. Romanova, \& G. S. BisnovatyiKogan, Mon. Not. R. Astron. Soc., 275, 244 (1995)

[55] D. A. Uzdensky, Astrophys. J., 620, 889 (2005)

[56] H. Li, R. V. E. Lovelace, J. M. Finn, \& S. A. Colgate, Astrophys. J., 561, 915 (2001)

[57] Y. Kato, M. R. Hayashi, \& R. Matsumoto, Astrophys. J., 600, 338 (2004)

[58] Y. Kato, S. Mineshige, \& K. Shibata, Astrophys. J., 605, 307 (2004)

[59] Y. Kato, "Magnetic-Tower Jet Solution for Launching Astrophysical Jets", accepted to Astrophys. \& Space Sci. (2006)

[60] M. Nakamura, H. Li, \& S. Li, Astrophys. J., 652, 1059 (2006)

[61] M. Nakamura, H. Li, \& S. Li, Astrophys. J., 656, 721 (2007)

[62] A. Ciardi, S. V. Lebedev, A. Frank, et al., "The evolution of magnetic tower jets in the laboratory", accepted to Phys. Plasmas (2007); e-print (astro-ph/0611441) 
[63] S. Hsu \& P. M. Bellan, Mon. Not. R. Astron. Soc., 334, 257 (2002)

[64] S. V. Lebedev, A. Ciardi, D. J. Ampleford, et al., Mon. Not. R. Astron. Soc., 361, 97 (2005)

[65] M. Nakamura, \& D. L. Meier, Astrophys. J., 617, 123 (2004)

[66] A. Königl, \& A. R. Choudhuri, Astrophys. J., 289, 173 (1985)

[67] A. Gruzinov, "Flares on the Black Holes", submitted to Astrophys. J. (1999)

[68] A. Tomimatsu, T. Matsuoka, \& M. Takahashi, Phys. Rev. D., 64, 123003 (2001)

[69] D. Eichler, Astrophys. J., 419, 111 (1993)

[70] M. C. Begelman, Astrophys. J., 493, 291 (1998)

[71] A. P. Goodson, K.-H. Böhm, \& R. M. Winglee, Astrophys. J., 524, 142 (1999)

[72] D. A. Uzdensky, Astrophys. \& Space Sci., 292, 573 (2004)

[73] D. Biskamp, Phys. Fluids, 29, 1520 (1986)

[74] D. A. Uzdensky \& R. M. Kulsrud, Phys. Plasmas, 7, 4018 (2000)

[75] R. M. Kulsrud, Earth, Planets and Space, 53, 417 (2001)

[76] L. M. Malyshkin, T. Linde, \& R. M. Kulsrud, Phys. Plasmas, 12, 102902 (2005)

[77] H. Ji, M. Yamada, S. Hsu, R. Kulsrud, T. Carter, \& S. Zaharia, Phys. Plasmas, 6, 1743 (1999)

[78] P. A. Sweet, in IAU Symp. 6, Electromagnetic Phenomena in Cosmical Physics, ed. B. Lehnert, (Cambridge: Cambridge Univ. Press), 123 (1958)

[79] E. N. Parker, J. Geophys. Res., 62, 509 (1957)

[80] H. E. Petschek, AAS-NASA Symposium on Solar Flares, (National Aeronautics and Space Administration, Washington, DC, 1964), NASA SP50, 425 (1964)

[81] M. Lyutikov, \& E. G. Blackman, Mon. Not. R. Astron. Soc., 321, 177 (2001)

[82] A. Soderberg, "Gamma-Ray Bursts in the Swift Era, Sixteenth Maryland Astrophysics Conference" Eds. Holt, S. S., Gehrels, N. \& Nousek, J. A., AIP Conference Proceedings, 838, 380 (2006)

[83] Y. Kaneko, E. Ramirez-Ruiz, J. Granot, et al., Astrophys. J., 654, 385 (2007)

[84] J. P. U. Fynbo, D. Watson, C. C. Thöne, et al., Nature, 444, 1047 (2006)

[85] M. Della Valle, G. Chincarini, N. Panagia, et al., Nature, 444, 1050 (2006)

[86] A. Gal-Yam, D. B. Fox, P. A. Price, et al., Nature, 444, 1053 (2006)

[87] A. I. MacFadyen, "Gamma-ray Burst and Afterglow Astronomy: 2001" AIP Conference Proceedings, 662, 202 (2003)

[88] E. G. Blackman, A. Frank, \& C. Welch, Astrophys. J., 546, 288 (2001)

[89] M. M. Romanova, G. V. Ustyugova, A. V. Koldoba, \& R. V. E. Lovelace, 2004, Astrophys. J., 616, L151 (2004)

[90] S. Komissarov, Mon. Not. R. Astron. Soc., 367, 19 (2006)

[91] J. C. McKinney, Mon. Not. R. Astron. Soc., 368, L30 (2006)

[92] A. Spitkovsky, Astrophys. J., 648, L51 (2006)

[93] S. Koide, K. Shibata, \& T. Kudoh, Astrophys. J., 522, 727 (1999)

[94] C. F. Gammie, J. C. McKinney, \& G. Tóth, Astrophys. J., 589, 444 (2003)

[95] L. Del Zanna, N. Bucciantini, \& P. Londrillo, Astron. \& Astrophys., 400, 397 (2003)
[96] J.-P. De Villiers, J. F. Hawley, \& J. H. Krolik, Astrophys. J., 599, 1238 (2003)

[97] P. Anninos, P. C. Fragile, \& J. D. Salmonson, Astrophys. J., 635, 723 (2005)

[98] S. S. Komissarov, Mon. Not. R. Astron. Soc., 359, 801 (2005)

[99] K.-I. Nishikawa, G. Richardson, S. Koide, K. Shibata, T. Kudoh, P. Hardee, \& G. J. Fishman, Astrophys. J., 625, 60 (2005) 


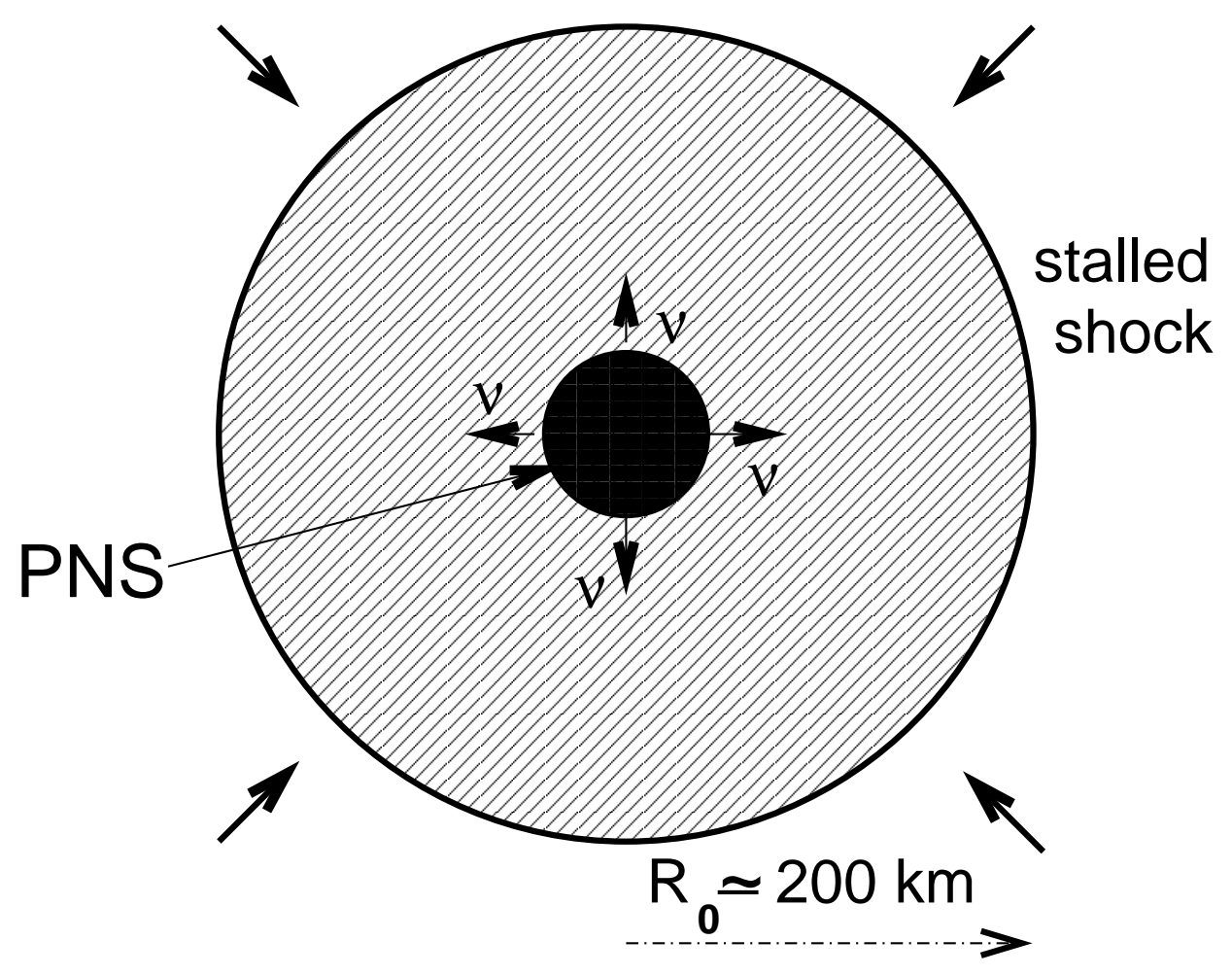

FIG. 1: Stalled shock phase of core-collapse explosion. 


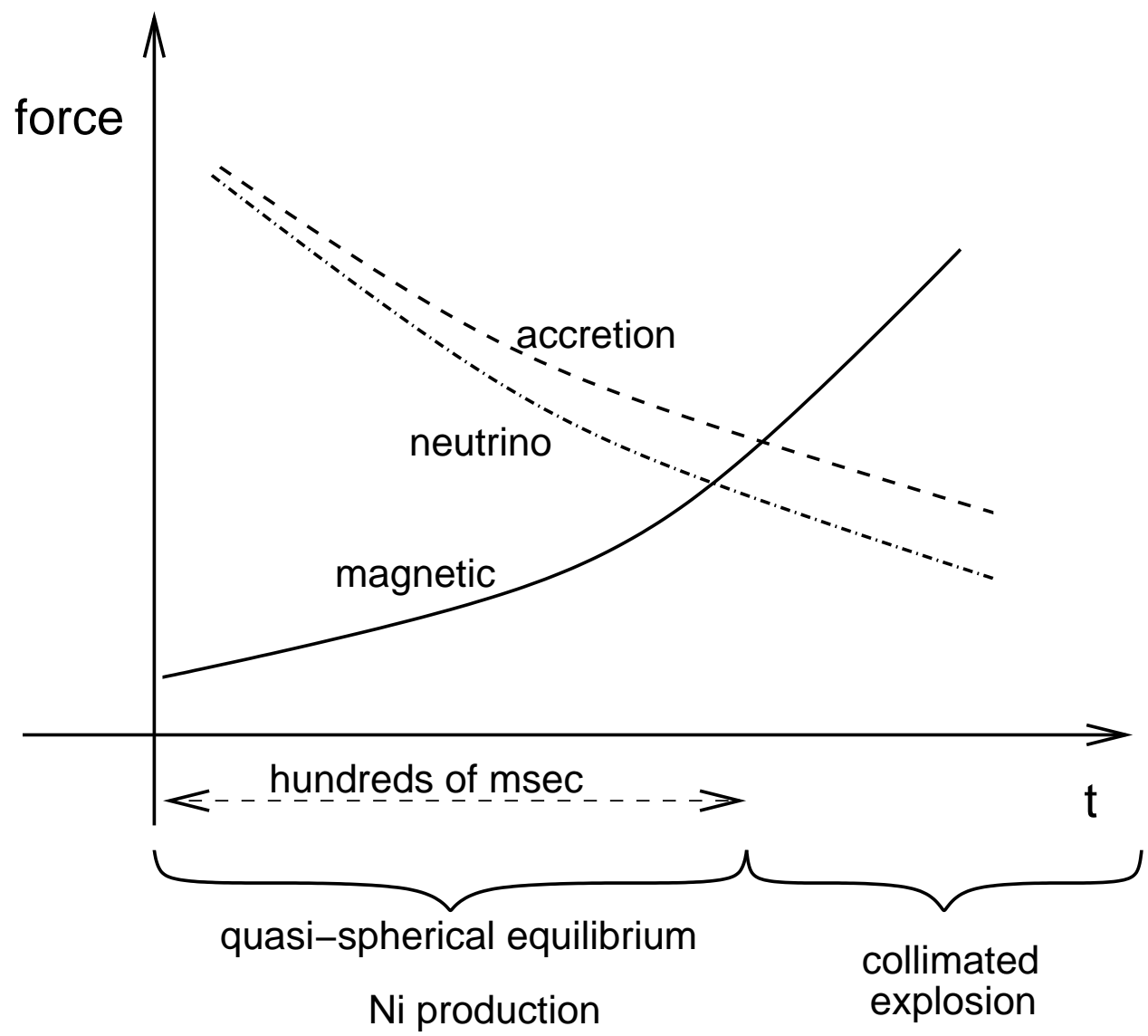

FIG. 2: Schematic time evolution of the main three forces responsible for the stalled-shock force balance. 


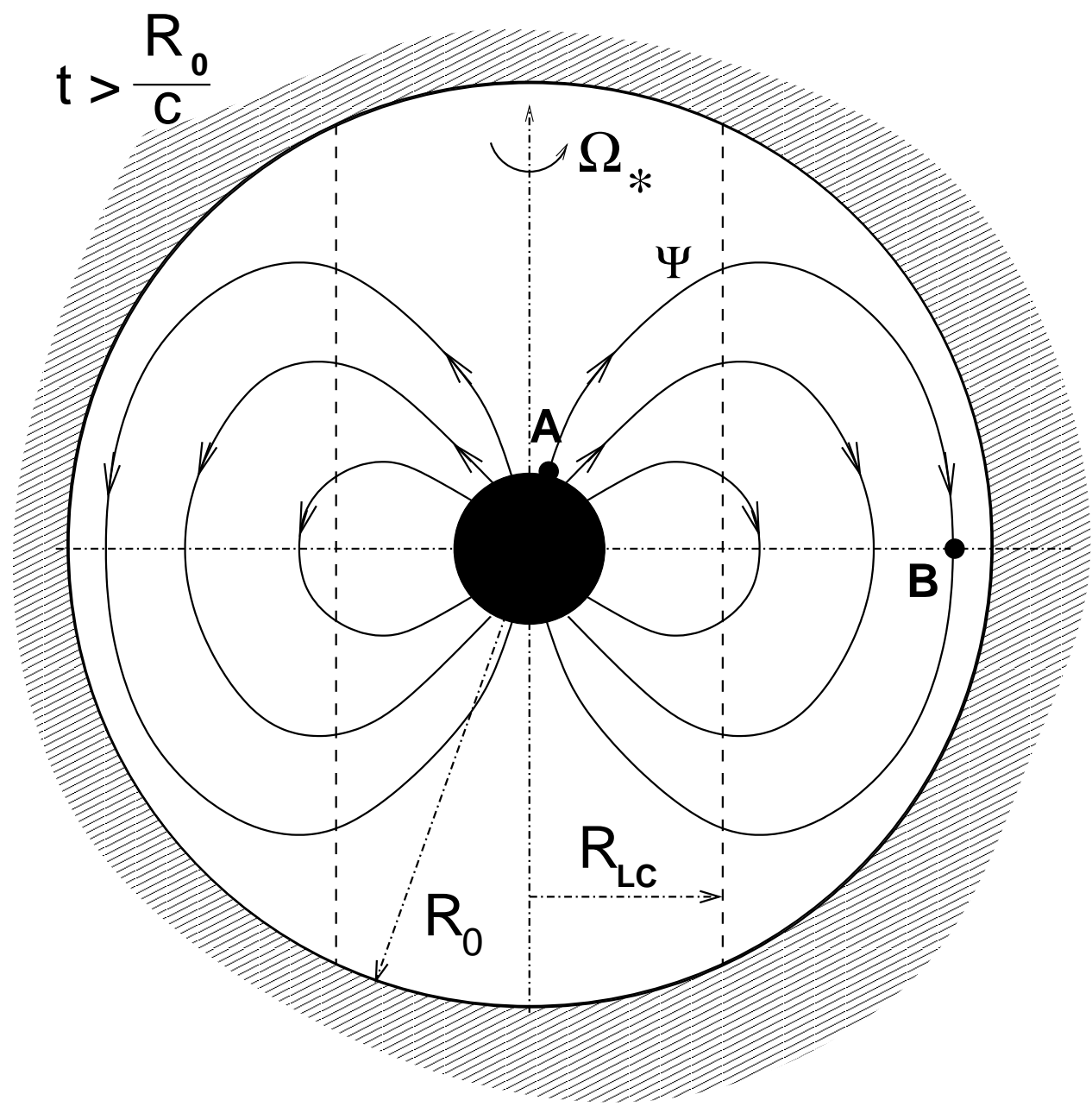

FIG. 3: Aligned pulsar inside an infinitely-conducting spherical cavity of radius $R_{0}$. The vertical dashed lines represent the pulsar's light cylinder of radius $R_{\mathrm{LC}}<R_{0}$. After a time of order the light-crossing time $R_{0} / c$, the poloidal field lines outside the light cylinder expand somewhat but still remain confined within the cavity. Because the toroidal magnetic field has to vanish at the equatorial midplane due to reflection symmetry, the field lines there cannot corotate with the star, $\Omega_{B}<c / R_{B}<\Omega_{*}$. As a result, differential rotation is established in both hemispheres, $\Delta \Omega=\Omega_{*}-\Omega_{B} \simeq \Omega_{*}$ (for $R_{B} \gg R_{\mathrm{LC}}$ ), which leads to continuous generation of toroidal magnetic flux. 


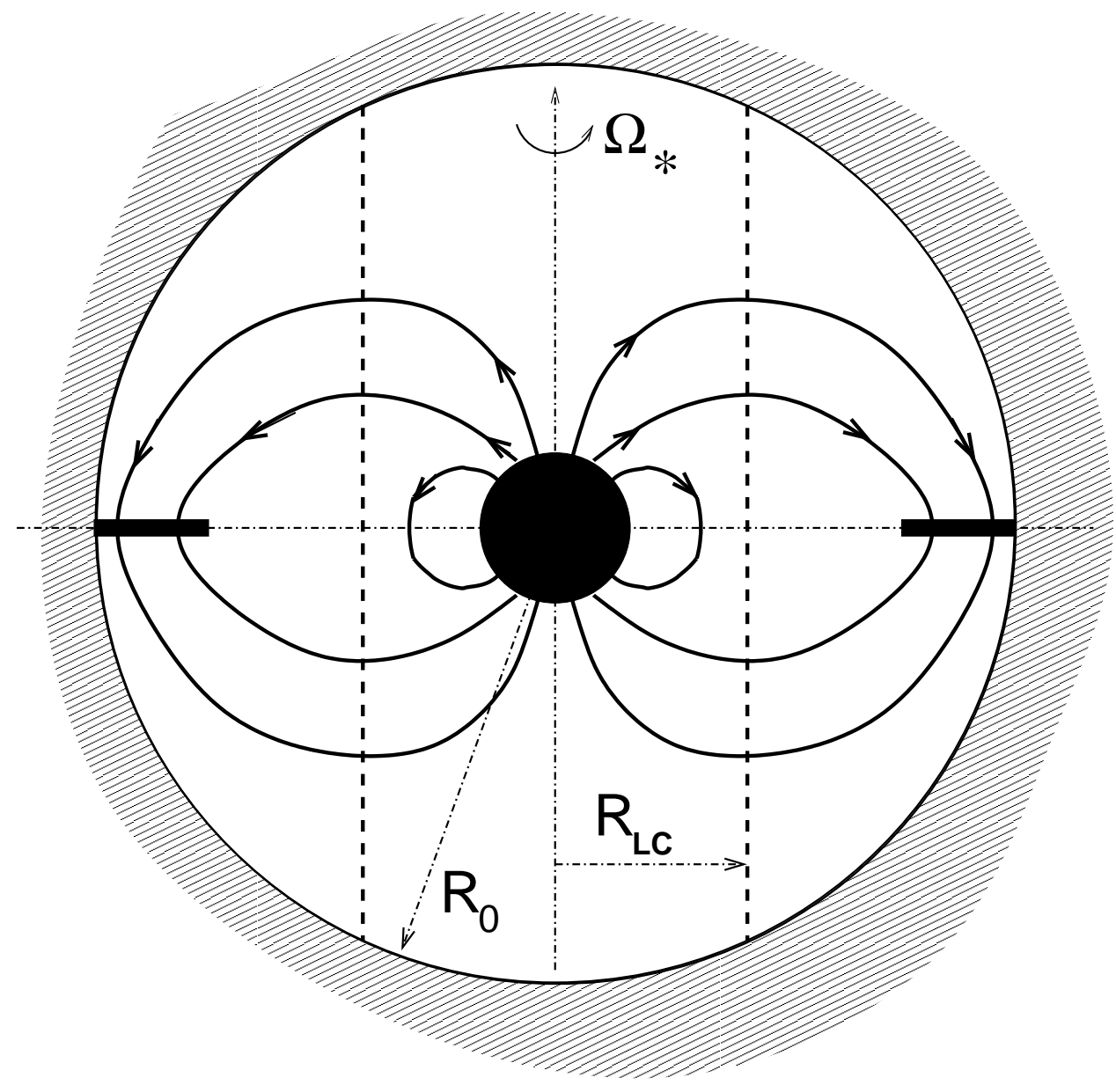

FIG. 4: At late times, the poloidal magnetic field is pressed against the wall by the centrifugal force of the rotating massive equatorial sheet. 


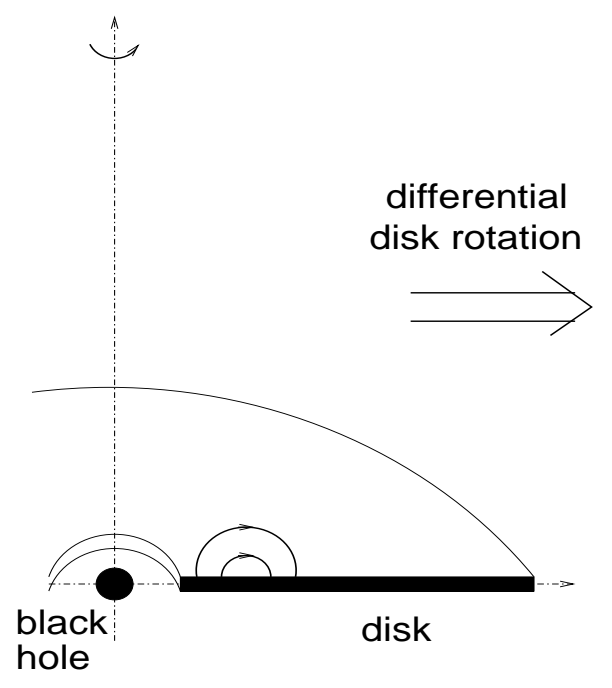

initial potential field

(a)

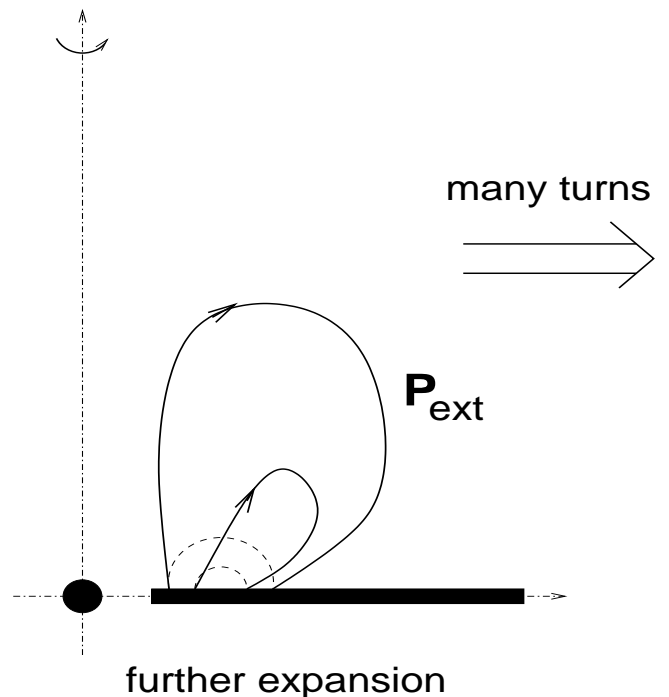

is vertical

(c)

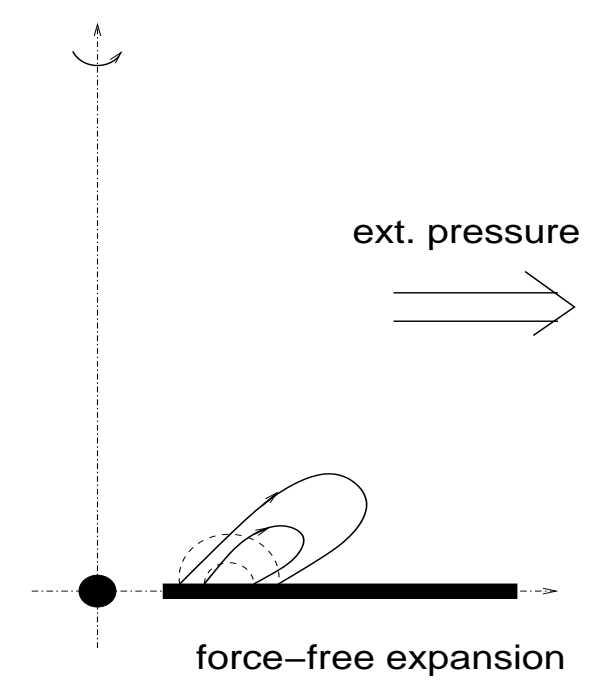

(b)

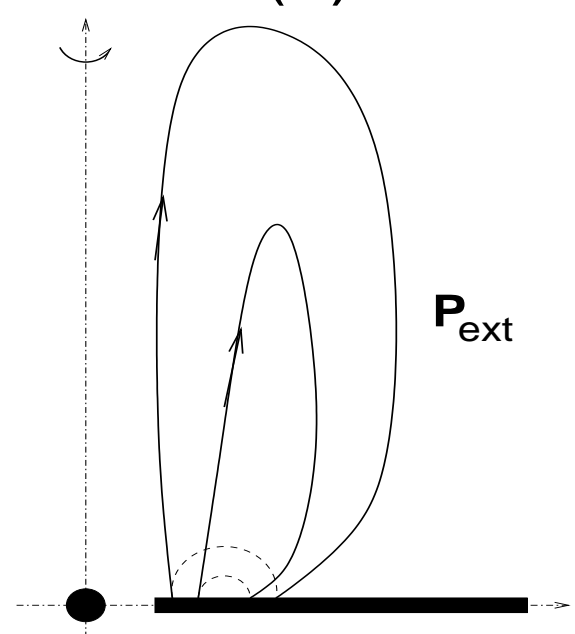

tall slender tower

(d)

FIG. 5: Development of a magnetic tower in Lynden-Bell's (1996) model. Reproduced from Ref. [15] by permission of the $A A S$. 


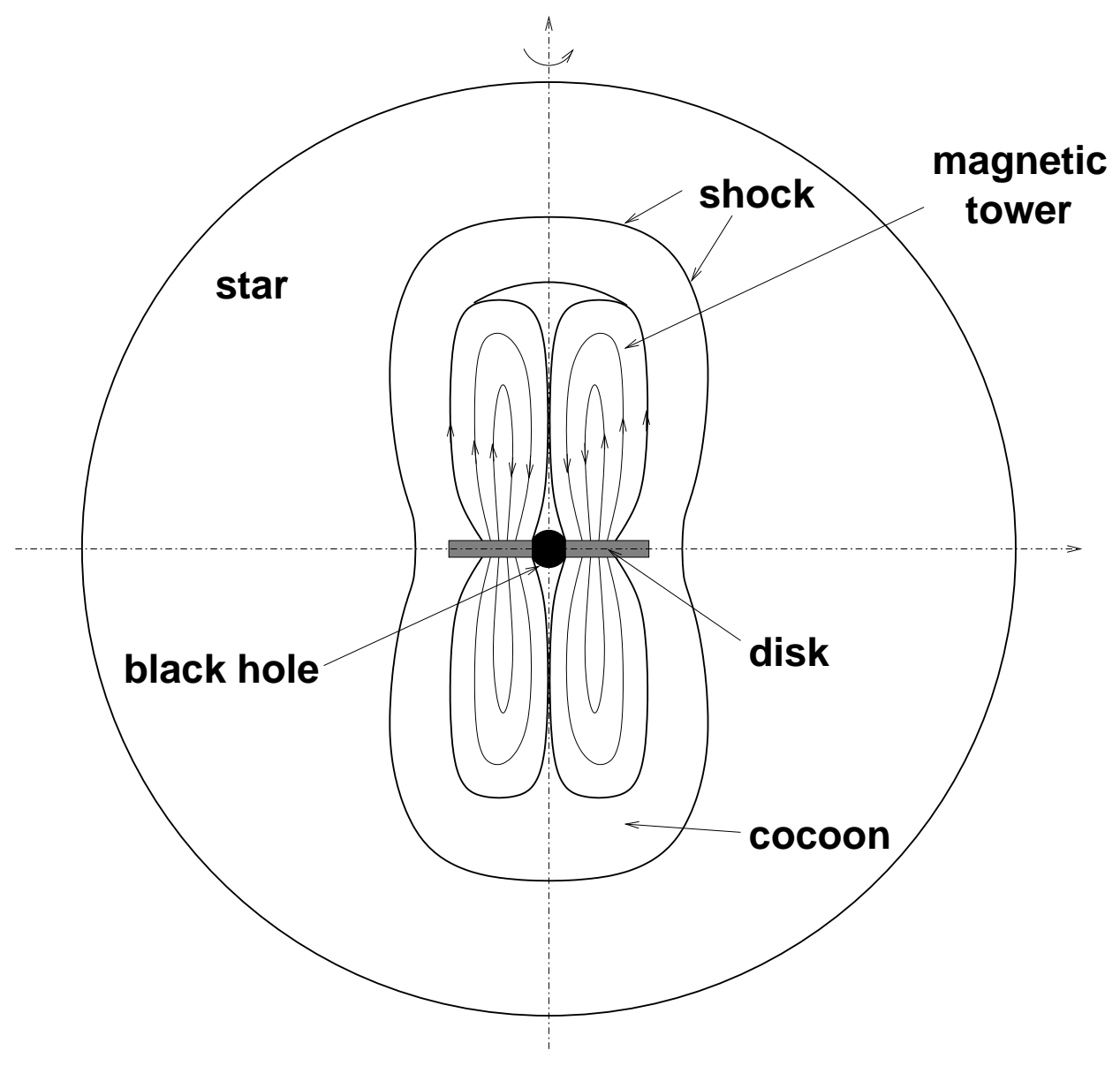

FIG. 6: Main components of a magnetic tower inside. The tower grows rapidly and drives a strong shock through the star. The shocked stellar gas behind the shock forms a hot cocoon whose high pressure confines the tower. Reproduced from Ref. [15] by permission of the AAS. 


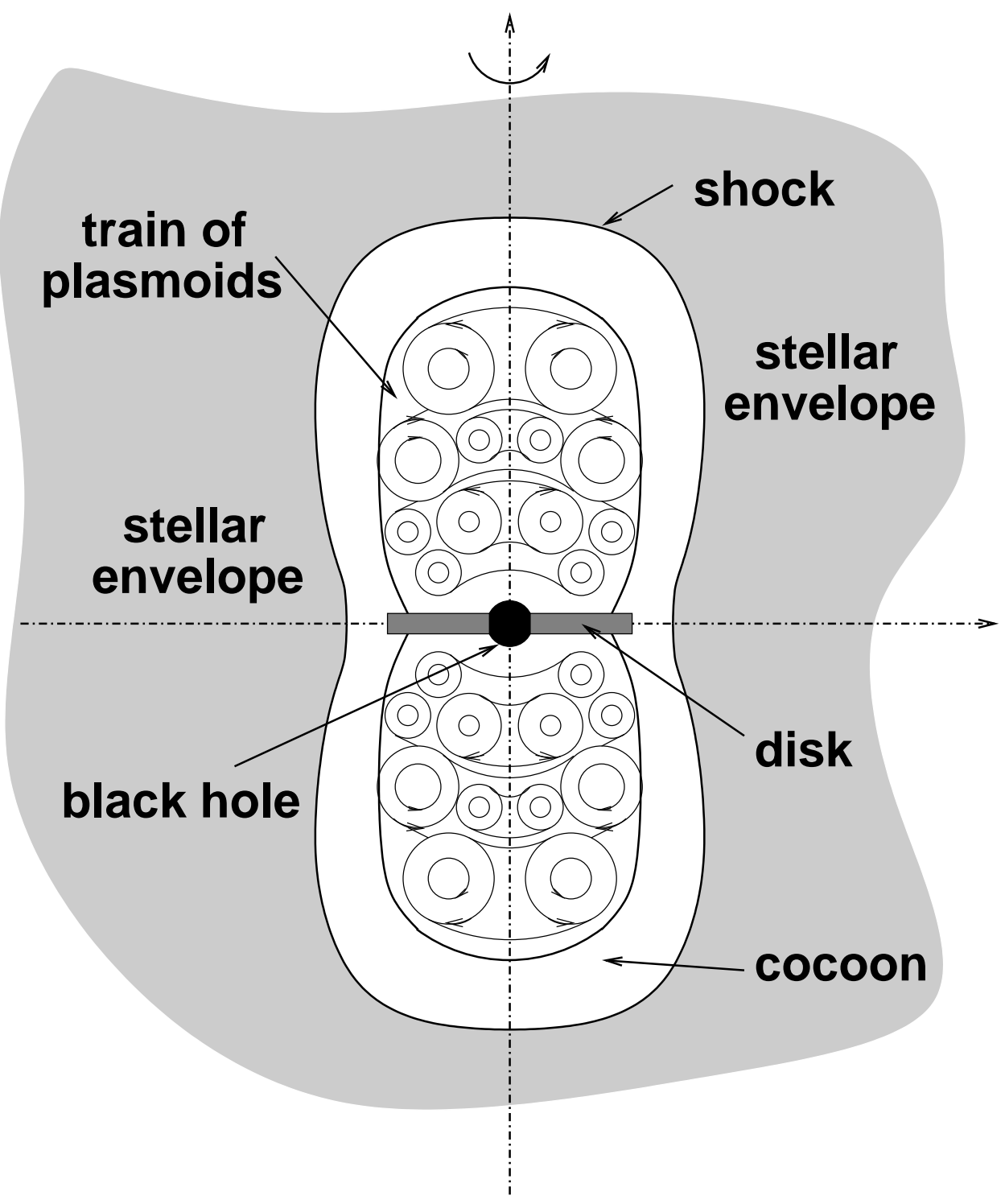

FIG. 7: Magnetic tower may have a substructure represented by a train of many spheromak-like plasmoids. This situation may arise as a result of spatial and temporal intermittency at the base of the outflow and/or due to instabilities and reconnection in the tower. 